\title{
1 In pursuit of biomarkers for predicting susceptibility to activity-based anorexia \\ 2 (ABA) in adolescent female rats
}

3

4 Laura K Milton $(\mathrm{PhD})^{1,2}$, Timothy Patton $(\mathrm{PhD})^{3,4}$, Meredith O’Keeffe $(\mathrm{PhD})^{3}$, Brian J Oldfield $5 \quad(\mathrm{PhD})^{1,2} \&$ Claire J Foldi $(\mathrm{PhD})^{1,2 *}$

6

$7 \quad{ }^{1}$ Monash University, Department of Physiology, 26 Innovation Walk, Clayton, 3800 Australia

$8 \quad{ }^{2}$ Monash Biomedicine Discovery Institute, 23 Innovation Walk, Clayton, 3800 Australia

$9{ }^{3}$ Monash University, Department of Biochemistry and Molecular Biology, Building 77 Wellington 10 Road, Clayton 3800 Australia

$11{ }^{4}$ Current address: University of Melbourne, Department of Microbiology and Immunology, The Peter Doherty Institute for Infection and Immunity, Elizabeth Street Melbourne, 3000 Australia

$13{ }^{*}$ Correspondence: Dr Claire J Foldi, Department of Physiology, Monash University, 26 Innovation 14 Walk, Clayton VIC 3800 Australia. E-mail: claire.foldi@monash.edu. Telephone +61(0)399059158

16 Running title: "Predictors of activity-based anorexia in rats" 


\section{Abstract}

Anorexia nervosa (AN) has high rates of mortality and low rates of recovery, with outcomes that worsen with illness duration. Improved early intervention strategies are required and identifying risk factors that contribute to the development of $\mathrm{AN}$ is critical to their implementation. The development of AN often follows a pre-existing diagnosis of anxiety disorders and obsessivecompulsive disorder and substantial genetic overlap between these conditions suggest common underlying features may predict vulnerability to AN. Moreover, patients with AN have increased levels of circulating proinflammatory cytokines, which may be involved in susceptibility to pathological weight loss considering that children with immune dysfunction have a higher risk of subsequent AN diagnoses.

Here, we used the activity-based anorexia ( $A B A$ ) model to examine whether baseline levels of locomotion, anxiety-like behaviour, compulsive behaviour, and circulating immune markers predict the subsequent development of pathological weight loss in adolescent female rats. While none of these primary features were shown to differentiate rats that went on to be susceptible or resistant to weight loss in $A B A$, increased locomotion and anxiety-like behaviour were both associated with the extent of weight loss in susceptible but not resistant animals. Intriguingly, behaviour related to poor decision-making in a situation of conflict was shown to predict vulnerability to ABA. Future research using the ABA model to uncover early predictors of AN should focus on translationally relevant assays of decision-making and cognitive behaviour, dysfunction of which may not only predispose animals to ABA but may also represent an endophenotype linking anorectic, anxietylike and compulsive behaviour. 


\section{Introduction}

Anorexia nervosa (AN) is a devastating condition with the highest mortality rate of any psychiatric disorder [1]. Up to fifty percent of patients with AN never recover [2], highlighting the urgent need for more effective therapeutic strategies. Recovery from AN becomes much less likely the longer the illness has persisted [3], therefore, early detection of symptoms and intervention are necessary for improving treatment outcomes [4]. Identifying biomarkers to help predict AN risk and/or symptom severity will enable treatment to be directed at prodromal symptoms, before weight loss becomes too severe and protracted. Both comorbid diagnoses and the expression of symptoms related to depression, anxiety and obsessive-compulsive disorder (OCD) are associated with worsened illness, poorer treatment outcomes, and increased relapse in patients with AN [5-9]. There is evidence to suggest that the development of AN often follows a pre-existing diagnosis of these comorbid conditions [10-15], presenting a potential target for early intervention strategies. However, a diagnosis of $\mathrm{AN}$ can also precede the development of, or be determined coincident with, comorbid psychiatric disorders [10, 13, 14], making it difficult to dissect the nature of the interaction between AN and depressive, anxious, and obsessive/compulsive symptoms.

In support of the hypothesis that anxiety and obsessional behaviour may increase susceptibility to AN in vulnerable individuals, childhood temperaments and personality traits including "picky eating", perfectionism, harm avoidance and rigid patterns of thought and behaviour $[16,17]$, related to levels of anxiety and compulsiveness [10], have been associated with the subsequent development of AN. These traits often become exacerbated in the acute stage of AN and improve with weight recovery but remain elevated compared to healthy controls $[10,18]$, indicating that underlying personality traits are partially independent of illness status and may predispose individuals to developing AN. Moreover, there are increased diagnoses of depression, anxiety, and OCD (as well as the non-pathological symptoms of these conditions) in healthy first-degree relatives of patients with AN compared to the general population [19-21] and substantial genetic overlap between AN and generalized anxiety disorder [22], major depressive disorder [23] and OCD [24]. Taken together, these findings suggest the existence of heritable risk factors for multiple 
psychiatric diagnoses that cluster together and sometimes result in the development of AN. This is not surprising considering that each of these mood-related pathologies are associated with common neurobiological and behavioural phenotypes with AN [25-27].

While association of mood disorders and AN as outlined above are often established in a post hoc manner, physiological correlates should provide earlier and more definitive predictors of subsequent susceptibility to AN. In this regard, disturbances in immune function may play a causal role in the development of $\mathrm{AN}$, considering that children and adolescents with autoimmune and autoinflammatory disorders are shown to have a higher risk of subsequent AN diagnoses [28] and genes associated with various immune-related phenotypes including asthma, vitiligo and type-1 diabetes are associated with AN [29]. Adults with AN have a distinct pattern of immune mediator expression, the most consistently documented of which are increased levels of the inflammatory markers RANTES (regulated upon activation, normal T cell expressed and secreted) [30], tumour necrosis factor-alpha (TNF-a) and interleukin-6 (IL-6) [31]. Pro-inflammatory cytokines produced both centrally and peripherally can have profound effects on brain function and behaviour relevant to food intake [32] and have been shown to remain elevated after weight recovery in AN patients [33]. However, the notion whereby immune markers might be used to screen for susceptibility to AN should be balanced by evidence suggesting that elevated proinflammatory cytokine expression in patients with AN is ameliorated with weight restoration [34], consistent with at least some of these changes being consequences of the conditions of chronic malnutrition typical of AN. This may include effects of a "leaky gut" induced by an anorectic state, whereby increased intestinal permeability allows antigens to traverse the intestinal wall and infiltrate systemic circulation, contributing to chronic, low-grade inflammation [35]. Intriguingly, increased levels of the same proinflammatory cytokines (IL-6 and TNF- $\alpha$ ) are seen in patients with OCD [36] and generalised anxiety disorder is also associated with an inflammatory response that includes increased TNF- $\alpha$ [37]. These conditions are not associated with chronic undernutrition but, as described above, are both commonly comorbid with $\mathrm{AN}$ diagnoses, raising the potential that there are common inflammatory mechanisms involved in the aetiology of these disorders. 
92

93

94

95

96

97

In order to determine behavioural and immune factors that contribute to the aetiology of AN, animal models are required. The most robust and widely-used animal model of AN is known as activitybased anorexia (ABA), which allows for a detailed interrogation of the biological mechanisms underlying pathological weight loss that is prohibited by the retrospective or cross-sectional nature of human studies. The ABA model pairs unhindered access to running wheels with time-limited food access and recapitulates the core features of the human condition including rapid body weight loss, voluntary hyperactivity and voluntary reductions in food intake [38-40]. We have previously shown that pathological weight loss does not occur in all rats exposed to ABA conditions, and in fact a subpopulation of adolescent female Sprague-Dawley rats remain resistant to weight loss, even when exposed to the same experimental conditions [41]. These susceptible and resistant subpopulations have been replicated in other studies investigating the neurobiology of AN in ABA rats [42] and mice [43], supporting the use of the ABA model for examining behavioural and immune phenotypes that predispose animals to pathological weight loss.

Exposure to ABA conditions is shown to increase anxiety-like behaviour on the elevated plus-maze (EPM) and the open field (OF) test [44-46], however, much like human studies, it is unclear whether this a consequence of weight loss or involved in vulnerability to ABA. Anxiety-like behaviour has been examined after only a single day of restricted food access in ABA mice, prior to excessive weight loss, and shown to be negatively correlated with excessive wheel running but not associated with body weight [47]. Only one study to date has examined how immune function is altered in the ABA model, with increased expression of interleukin-10 (IL-10), TNF- $\alpha$ and interleukin-1 $\beta$ (IL-1 $\beta$ ) observed in the colonic mucosa of ABA mice compared to mice subject to the same food restriction paradigm without access to a wheel, indicating an effect specific to ABA rather than the result of limited food intake [48]. This was accompanied by elevated levels of IL-1 $\beta$ and its receptor (interleukin-1 receptor-1; IL-1R1) in the hypothalamus of ABA mice, although hypothalamic IL-1 $\beta$ expression was also increased in mice with restricted food access compared to controls. ABA exposure in this study was also shown to increase intestinal permeability, measured by circulating levels of zonulin, a plasma marker of gut barrier that modulates intercellular tight junctions [49]. Increased zonulin following ABA could therefore contribute to a "leaky gut" allowing 
120

121

122

123

124

125

126

127

128

129

130

131

132

133

134

135

136

137

138

139

140

141

142

143

proinflammatory molecules into circulation. It is plausible that early activation of the intestinal immune response could trigger systemic low-grade inflammation during $A B A$, however it remains unknown whether immune disturbances might predispose animals to developing the $A B A$ phenotype.

In this study, we aimed to determine whether levels of anxiety-related and compulsive behaviour in rats could be used to predict susceptibility to pathological weight loss under ABA conditions, and whether differences in proinflammatory cytokine expression correlated with behaviour or the development of ABA. Our goal was to identify markers for the development of anorectic behaviour in a well-established animal model that could be used to screen at-risk individuals in order to direct early intervention strategies. This objective is in keeping with broader efforts to define predictive markers of future susceptibility to ABA in rodents and $A N$ in humans that encompass not only behavioural but biological traits.

\section{Materials and methods}

\section{Animals}

Female Sprague-Dawley rats $(n=44)$ were sourced from the Monash Animal Research Platform (MARP; Clayton, VIC, Australia) at 6 weeks of age with initial body weights between 120-160g. To determine whether baseline locomotor activity, anxiety-like or compulsive behaviour predicted susceptibility to weight loss in ABA, rats were tested on separate and consecutive days on the elevated plus maze (EPM), open field test (OF) and marble burying test (MBT) before exposure to ABA conditions. Blood samples were taken before behavioural testing and after recovery from the ABA paradigm to screen for proinflammatory cytokines. All experimental procedures were approved by the Monash Animal Resource Platform Ethics Committee (project ID:15171) and are detailed in Fig 1. A singly housed male rat was present in each experimental room to synchronise the estrous cycles of the female rats (known as the Whitten effect; [50]). 
144

145

146

147

\section{8}

149

150

151

152

153

154

155

156

157

158

159

160

161

162

163

164

165

166

167

168

\section{Behavioural testing}

Maze-based behavioural tests were recorded with an overhead camera connected to a computer and analyzed with TopScan Lite tracking software (V 2.0; CleverSys VA, USA) and manual scoring by an observer blinded to experimental condition.

Anxiety-like behaviour and general locomotor activity

The elevated plus maze (EPM) consisted of an elevated 4-arm platform made of grey perspex (70 $\mathrm{cm}$ long $\times 10 \mathrm{~cm}$ wide $\times 90 \mathrm{~cm}$ high) with two closed (40 cm high walls) and two open arms. Rats were placed in the centre platform $(10 \times 10 \mathrm{~cm})$ facing an open arm and the proportion of time spent in the closed arms relative to the open arms in each $10 \mathrm{~min}$ trial, was used as the primary measure of anxiety-like behaviour [51]. Frequency of head-dipping and rearing behaviour was also recorded

to assess aspects of exploration. The open field (OF) test consisted of a deep open topped box (60x60x55 cm deep) in which distance travelled in each $10 \mathrm{~min}$ trial was used as the primary measure of locomotor activity [52] and the proportion of time spent in the aversive centre zone (middle square of a $3 \times 3$ grid; $20 \times 20 \mathrm{~cm}$ ) was used as a secondary measure of anxiety-related behaviour [53]. Assesment of vertical exploration was obtained during this test by recording the frequency of rearing.

\section{Compulsive behaviour}

The marble burying test (MBT) consisted of the same OF box described above with a $5 \mathrm{~cm}$ deep layer of sawdust bedding on which 9 marbles (blue glass; $8 \mathrm{~mm}$ diameter) were placed in an even grid pattern. The "classical" measure of compulsiveness in the MBT is number of marbles buried at the end of the test duration (in this case $10 \mathrm{~min}$ ), where a greater number indicates increased compulsive tendencies [54, 55]. However, this measure does not account for the likelihood that rats will bury and unbury marbles throughout the test, and produce a confounding ceiling effect [56], therefore the number of interactions with marbles (including burying and unburying) was used as the primary measure of compulsive behaviour in this study. 
170 Four experimental groups were used to examine the influence of behavioural and immune factors

171 on susceptibility to $A B A$, and rats were allocated to groups to ensure an even spread of starting

172 body weights. Animals either underwent exposure to the ABA paradigm (ABA; $n=20$ ), had ad

173 libitum access to food paired with access to a running wheel (RW; $n=8$ ), had time-limited food 174 restriction with no wheel access (FR; $n=8)$, or ad libitum food access and no running wheel 175 (control; $n=8$; see Fig 1). Twenty rats were allocated to the ABA conditions to allow for the 176 emergence of Susceptible and Resistant populations with adequate numbers in each. ABA and 177 RW groups were individually housed in transparent activity wheel and living chambers (Lafayette 178 Instruments, IN, USA; model 80859) whereas FR and control groups were singly housed in 179 standard wire topped polypropylene cages. All groups were housed in a temperature $\left(22-24^{\circ} \mathrm{C}\right)$ 180 and humidity (30-50\%) controlled room under a reversed $12 \mathrm{~h}$ light/dark cycle (lights off at $1400 \mathrm{~h}$ ). 181 Food restriction lasted for a maximum of 10 days and ABA conditions persisted until rats reached $182 \leq 80 \%$ baseline body weight, on the second day of spontaneous weight gain or for a maximum of 18310 days, whichever occurred first, at which point ad libitum access to food was reinstated. Body 184 weight and food intake were recorded daily between 1330h-1400h over the 10-day experimental 185 period, with 90 min food intake also recorded at $1530 \mathrm{~h}$.

187 Each running wheel was equipped with an Activity Wheel Counter (Lafayette Instruments, IN, 188 USA), which was connected by USB interface to a computer running Activity Wheel Software 189 (Lafayette instruments). Running wheel activity (RWA) was recorded in 10-minute intervals and 190 RWA in the hour before feeding 1300-1400h was used to assess food anticipatory activity (FAA). 191 To control for individual differences in the propensity to run, FAA was calculated as a proportion of 192 total daily RWA and food-restriction evoked hyperactivity was calculated for individual rats as the 193 change in running from baseline to $A B A$ (ABA-Baseline). 
194 $\underline{\text { Plasma collection and cytokine analysis }}$

195

Blood was collected from tail tip at 6 weeks of age, 5 days prior to the commencement of

196 behavioural testing. Approximately 350 ul of whole blood was collected into EDTA coated tubes

197 (Microvette; Brand), which were centrifuged for $10 \mathrm{~min}\left(8000 \mathrm{rpm}, 4^{\circ} \mathrm{C}\right)$ within $30 \mathrm{~min}$ of collection 198 and plasma separated and stored at $-80^{\circ} \mathrm{C}$ until use. Following exposure to ABA conditions, 7 199 days of ad libitum food access and body weight recovery to $>100 \%$ baseline, blood was collected 200 via cardiac puncture. A custom rat multi-analyte LEGENDplex bead-based immunoassay kit was 201 202 203 204 205 206 used to examine cytokine concentrations in plasma samples (LEGENDplex; BioLegend, CA, USA) that targeted six cytokines concurrently (IL-6, IL-10, IL-4, IL-1 $\beta$, TNF- $\alpha$, RANTES). These analytes were selected based on their previously reported elevation in human AN patients and/or ABA mice. Plasma samples were screened with the LEGENDplex assay kit as per manufacturer's instructions, and the readout measurement acquired using a Fortessa X-20 flow cytometer (Becton Dickinson (BD); Franklin Lakes, NJ, USA). Data were analysed using LEGENDplex Data Analysis Software (v8.0; BioLegend).

\section{$\underline{\text { Statistical analyses }}$}

Except otherwise noted all statistical analysis was performed in GraphPad Prism 8.0 (GraphPad Software, San Diego, CA). Significance for all tests was set at $p<0.05$. A variety of statistical analyses were used determined by the type of data and number of groups: One- and Two-way ANOVA, followed by post-hoc multiple comparisons (Tukey's or with a Bonferroni correction) when applicable; independent samples t-test; Pearson's correlation; and linear regression. Details of each individual analysis and complete statistical results can be found in Supplementary Results.

\section{Exclusions}

One rat exposed to ABA showed an abnormal body weight loss trajectory and one rat exposed to FR was unable to maintain body weight $>80 \%$ of baseline; data collected from both these animals was excluded from all analyses. Concentrations of IL-10, TNF- $\alpha$ and IL-1 $\beta$ were not detected at levels high enough to be reliable using the LEGENDplex assay so these analytes were excluded 
220

221

222

223

224

225

226

227

228

229

230

231

232

233

234

235

236

237

238

239

240

241

242

243

244

245

from all analyses. Any pre- and post-exposure pair of RANTES, IL-4 or IL-6 samples that was incomplete was excluded from analyses.

\section{Results}

\section{Effects of individual components of the ABA paradigm on weight maintenance and feeding}

Of rats exposed to ABA conditions (Fig 2A), 9/19 (47\%) were resistant to body weight loss (Fig 2B), and while weight loss was negatively correlated with food intake for Resistant animals, in line with all three control groups (all $p s<.0001$ ), there was no such association for rats Susceptible to ABA ( $p=.1610$; Fig 2C). Moreover, the body weight loss trajectories for Resistant rats plateaued similarly to rats without access to a running wheel (FR; Fig 2D), whereas Susceptible rats lost significantly more body weight on average per day than both Resistant and FR rats (both $p s<.0001$; Fig 2E). Access to a running wheel alone did not produce significant weight loss (RW, $p=.1446$; Fig 2F) but did increase food intake compared to control rats ( $p=.0003$; Fig 2G and I) presumably to compensate for increased energy expenditure from wheel running. Much like the pattern of body weight loss, Resistant rats ate a similar amount of food to FR rats ( $p=.9982$; Fig 2G and $\mathbf{H}$ ) whereas Susceptible rats ate significantly less than both other food restricted groups (both ps<.0001; Fig 2G and $\mathbf{H})$.

\section{Running wheel activity predicts susceptibility to weight loss under ABA conditions}

Consistent with our previous findings [41], running activity in the two days prior to food restriction predicted susceptibility to pathological weight loss in ABA, and food restriction-evoked hyperactivity did not occur in animals resistant to weight loss (all ps<.0001; Fig 3B). Food restriction-evoked hyperactivity was especially pronounced in Susceptible rats during dark phase running (all $p s<.0063)$, but also occurred in the light phase ( $p=.0156$; Fig $3 \mathrm{C})$. This shift toward light phase running in Susceptible animals was evident by the third day of ABA, with the light-phase activity peak constituting almost all running activity in Susceptible rats by ABA Day 7 (Fig 3D). Resistant rats also increase light-phase running during $A B A$, but preferentially within the hour preceding food access $(p<.0001$; Fig 3E), a deliberate and motivated increase in anticipation of food (FAA). On 
246

247

248

249

250

251

252

253

254

255

256

257

258

259

260

261

262

263

264

265

266

267

268

269

270

271

272

average, there were no differences between groups during baseline running and Resistant rats ran

at levels similar to RW rats, whereas Susceptible rats show marked hyperactivity during ABA compared to both other groups (both $p s<.0001$; Fig 3F). When we examined the change in running activity elicited by $A B A$ in individual rats to account for baseline variability, hyperactivity remained specific for Susceptible rats compared to Resistant ( $p=.0022)$ and RW rats ( $p=.0008$; Fig 3G). Interestingly, daily running was positively correlated with body weight for RW ( $p=.0012)$ but not Resistant rats $(p=.1476$; Fig $\mathbf{H})$ and, conversely, associated with greater food intake for Resistant rats $(p<.0001)$ but not RW rats $(p=.0774$; Fig 3I) suggesting that while overall running did not differ between these groups, the relationship between running and other measures of energy balance did. The aspect of running that was specifically increased in Resistant rats, FAA, was elevated over Susceptible and RW levels as both a percentage of running activity during ABA (Fig $\mathbf{3} \mathbf{J}$ ) and change from baseline levels (all ps<.0019; Fig 3K), and was inversely associated with body weight loss in Resistant animals ( $p<.0001$; Fig $3 \mathrm{~L}$ ). FAA is considered to be a motivated behaviour that occurs in periods of food scarcity to increase the likelihood of finding food. It is therefore noteworthy that while FAA was positively associated with food intake for Resistant $(p<.0001)$ and RW ( $p=.0027)$ animals, this association was absent in rats susceptible to ABA ( $p=.9293$; Fig $3 \mathbf{M})$.

Measures of anxiety-like and compulsive behaviour do not predict subsequent susceptibility to ABA

The duration of time spent in different zones of the EPM (Fig 4A) did not differentiate between rats that went on to be susceptible or resistant to ABA ( $p=.9979$; Fig 4B); however, rats that went on to be susceptible to ABA entered and exited zones more often than rats that went on to be resistant to $\operatorname{ABA}(p=.0053$; Fig $4 \mathrm{C})$, exemplified by more frequent crosses into the centre zone $(p=.0355)$, without an overall increase locomotor activity ( $p=.1215$; Fig 4D). The number of exploratory rears ( $p=.6048$; Fig 4E) or head dips in the EPM ( $p=.2709$; Fig 4F) did not differ between rats that went on to be susceptible or resistant to ABA, however, the primary measure of anxiety-like behaviour on the EPM, duration spent in the open arms, was correlated with the extent of weight loss in ABA for Susceptible rats only ( $p=.0421$; Fig 4G). Similarly, while measures of locomotion, anxiety-like or exploratory behaviour in the OF (Fig $\mathbf{4 H}$ ) did not predict susceptibility to ABA (distance travelled 


\section{3}

274

275

276

277

278

279

280

281

282

283

284

285

286

287

288

289

290

291

292

293

294

295

296

297

298

$p=.8629$, duration in centre $p=.5724$, entries into centre $p=.6799$, rearing $p=.7875$; Fig 4I-L), locomotor activity was correlated with the extent of weight loss in Susceptible $(p=.0091)$ but not Resistant ( $p=.4891)$ rats (Fig $\mathbf{4 M}$ ). Of note, given that RWA at baseline was predictive of ABA susceptibility but general locomotor activity was not, there was also no relationship between total distance travelled in the OF and baseline RWA (Susceptible, $p=.1538$; Resistant, $p=.5278$; Fig 4N). Moreover, while there was a trend toward increased interactions with marbles in the MBT (Fig 40) for rats that went on to be resistant to $A B A(p=.0551$; Fig $4 P$ ), this measure was not correlated with running wheel activity at baseline or during $A B A$ (all ps>.1873; Fig 4Q) but was positively associated with food intake for Resistant $(p=.0305)$ but not Susceptible $(p=.1319)$ rats (Fig 4R). Considering that Resistant rats ate significantly more than Susceptible rats, this suggests that interactions with marbles may be less reflective of compulsive behaviour and perhaps represents increased exploration directed toward novelty (i.e. the introduction of marbles or food hoppers to the environment).

\section{Expression of pro-inflammatory cytokines does not predict susceptibility to ABA}

Expression of IL-10, IL-1 $\beta$ and TNF- $\alpha$ was below the assays limit of detection in a large proportion of samples tested, precluding reliable comparisons between groups (data not shown). Expression of RANTES (Fig $5_{\mathrm{B} 1-\mathrm{B} 3}$ ), IL-4 (Fig $5_{\mathrm{C} 1-\mathrm{C} 3}$ ) and IL-6 (Fig $\mathbf{5}_{\mathrm{D} 1-\mathrm{D} 3}$ ) was not significantly different between groups prior to exposure to the various experimental conditions, was not systematically altered by access to a running wheel or restricted food access and did not predict susceptibility or resistance to pathological weight loss under ABA conditions. However, baseline expression of IL-6 was positively associated with change in body weight for Resistant but not Susceptible rats ( $p=.0305$ and $p=.4627$ respectively; Fig $5 \mathbf{E}$ ), and the association between IL- 6 and running activity was in opposite directions for Susceptible and Resistant rats, with increased IL-6 associated with higher running activity for Susceptible $(p=.0404)$ but lower running activity for Resistant rats $(p=.0386$; Fig 5F). Moreover, the extent to which ABA elicited a change in IL-6 expression was associated with food intake only for Susceptible $(p=.0128)$ but not Resistant $(p=.9219)$ rats (Fig 
299

300

301

302

303

304

305

306

307

308

309

310

311

312

313

314

315

5G). Finally, the change in IL-4 expression elicited by ABA was associated with increased weight loss for Susceptible $(p=.0173)$ but not Resistant $(p=.8211)$ rats (Fig $5 \mathbf{H})$.

\section{Discussion}

Mood-related comorbidities and immune dysregulation both contribute to increased risk and severity of AN in human patients. Here, we show that neither the behavioural correlates of anxiety, locomotion and compulsivity nor circulating inflammatory markers that are associated with AN contribute to susceptibility to pathological weight loss in the ABA rat model. Contrary to expectation, classical measures of anxiety-like behaviour or general locomotor activity did not differentiate between rats that went on to be susceptible or resistant to ABA. However, specific aspects of the ABA microstructure were differentially associated with behavioural phenotypes in Susceptible versus Resistant rats, providing new insight into why pathological weight loss occurs in some animals exposed to ABA conditions but not others. For example, increased anxiety-like behaviour on the EPM and hyperlocomotion in the OF were associated with a greater extent of weight loss in ABA for Susceptible but not Resistant rats, suggesting that these behavioural features contribute to increased weight loss in Susceptible individuals. This is consistent with evidence that childhood or adolescent anxiety disorder symptoms are associated with lower BMI in individuals with AN [57].

Moreover, rats that went on to be susceptible to weight loss in ABA demonstrated a significant increase in the number of crossings between zones on the EPM, which was driven by more frequent centre crossings. The centre of the EPM is considered to be a point of choice or decisionmaking, from which animals engage in high levels of risk assessment [58]. Thus, if an animal is particularly indecisive about which arm to explore, the result is increased centre platform activity [59], which has been associated with inefficient responding in an operant conditioning task [60].

This suggests that susceptibility to weight loss in ABA in the present study was influenced by cognitive function, specifically poor decision-making. Deficits in cognitive capacities are common to patients both currently ill with and weight-recovered from AN [61-64], and cognitive function is 
325

326

327

328

329

330

331

332

333

334

335

336

337

338

339

340

341

342

343

344

345

346

347

348

349

350

351

impaired in rats after exposure to ABA [65]. We have recently shown a neurobiological link between pathological weight loss in ABA and cognitive flexibility using touchscreen-based assays of operant responding [66]. There is also evidence that cognitive dysfunction is a risk factor for general psychopathology in adolescents [67], and may therefore represent an endophenotype linking the comorbid development of mood disorders and AN. Future research should use translational assays of cognitive behaviour, such as those based in touchscreen technology, prior to exposure to ABA conditions in order to further interrogate this association.

This study also recapitulated our previous finding [41] that susceptibility to ABA could be predicted from baseline running wheel activity in the two days prior to initiation of food restriction, and built upon this to show that general locomotor activity was correlated with the extent of weight loss in Susceptible but not Resistant animals. However, the proportion of rats that were Resistant to developing the ABA phenotype in this study was $47 \%$ - a marked increase from the $~ 30 \%$ previously reported $[41,68]$. It is likely that the additional handling required for maze-based behavioural testing is responsible for this increase, considering that repeated postnatal handling reduces vulnerability to $A B A$ in adult rats [69]. It was also evident from the present study that locomotor and running activity were not correlated for any group of animals that had access to wheels. This discrepancy highlights the fact that general locomotion and activity in the running wheel are distinct behaviours, likely underpinned by different neurochemical and motivational processes. While general locomotor activity is a relatively constant behaviour, wheel running is a deliberate and driven behaviour requiring a conscious decision to engage in, and effort to perform [70]. Wheel running in ABA is also thought to be compulsive [71], however in the present study, RWA was not associated with any measure on the MBT and in fact there was a trend toward increased "compulsive" marble burying for rats that went on to be resistant to ABA. This indicates that either compulsive behaviour develops coincident with access to the wheel during $A B A$, rather than predisposes animals to pathological weight loss, or that the MBT is not a reliable measure of compulsivity in rats, but perhaps relates to investigative drive [72]. In support of the latter, marble burying was correlated with increased food intake for Resistant but not Susceptible rats, which may 
reflect an increased willingness to explore novel/changing environments (in this instance increasing approach to the food hopper and subsequent food intake when available).

354 With respect to how circulating proinflammatory cytokines might predict susceptibility to ABA, there 355 was no consistent change in expression of RANTES, IL-4 or IL-6 that was associated with any 356 aspect of the ABA paradigm when sampled at the baseline, pre-exposure state. This is perhaps 357 unsurprising considering changes in cytokine expression normally follow a "challenge", however, it 358 was interesting to see that IL-6 expression prior to exposure to ABA was associated with increased running activity for Susceptible rats, but decreased running activity for Resistant rats, highlighting a divergence between these populations. It is clear that the intensity and duration of exercise are key factors in determining changes in inflammatory cytokine expression, and that exercise-mediated inflammation presents a different effect depending on the tissue type examined. For example, chronic or exhaustive exercise in rats both increases cytokine concentrations in skeletal muscle and reduces concentrations in adipose tissue [73, 74], whereas moderate exercise reduces expression of inflammatory cytokines in skeletal muscle [75] and in serum [76]. Surprisingly, running activity appeared not to alter markers of inflammation in the present study, with no 367 significant changes in circulating cytokines elicited by either normal or excessive running behaviour. It is plausible that any changes in expression related to running activity may have been masked by opposing changes in tissue-specific release. Moreover, the effects of caloric restriction on pro-inflammatory cytokine production is similarly tissue-specific [77] but both intermittent and 371 chronic calorie restriction paradigms are shown to reduce serum IL-6 among other cytokines 372 compared to ad libitum feeding [78]. There were no significant changes in cytokine expression 373 elicited by food restriction in the present study, but the change in IL-6 expression following 374 exposure to ABA was associated with reduced food intake only for Susceptible rats. It should be 375 noted that post-exposure blood samples were collected after body weight recovery to at least 376 baseline levels, which also included a period of ad libitum food access for FR and ABA rats and no 377 running wheels for RW and $\mathrm{ABA}$ rats. Considering the points above, and that elevated 378 inflammatory markers seen in patients with AN have recently been shown to normalise with body 
weight recovery [34], it may be that a dysregulated inflammatory state is confined to the acute stage of anorexia in both humans and rats.

Despite some interesting hints that aspects of decision-making and anxiety-related behaviour could

382 be used to predict susceptibility to pathological weight loss in the ABA model, this study did not

383 identify a set of reliable behavioural or inflammatory features that predispose animals to ABA. It

384 may be premature, however, to exclude such predisposing behaviours on the basis of the tests

385 performed. These may lack the sensitivity or acuity to unveil underlying differences that will align

386 with susceptibility to ABA. Alternatively, there may be no single behavioural or immune-related

387 measure that predicts susceptibility to ABA, but rather a raft of small differences that independently

388 fail to predict susceptibility but, in concert, comprise a cumulative burden which promotes

389 susceptibility to ABA. This would fit with the difficulties in finding predictive markers for AN in

390 humans and also explain the deficiencies of current treatments that often target only a subset of

391 AN pathologies. It should be recognized that despite our intention to incorporate the most

392 appropriate behavioural tests that may highlight a predisposition, this list is not exhaustive and that

393 other predictive tests may be developed and utilised in the future, particularly with respect to

394 cognitive mediators of susceptibility to ABA. It may also be the case that traditional behavioural 395 testing, requiring extensive handling and experimenter intervention, is not the most appropriate 396 means by which to examine predisposing behaviours for ABA, considering that repeated handling 397 is known to alter susceptibility to ABA [69]. It is now possible with novel technologies to examine 398 naturalistic behaviour in rodent models without experimenter intervention, for example using fully 399 automated testing systems that rely on RFID technology to gate access of individual animals to 400 testing chambers $[79,80]$. Combining these automated systems with translationally relevant 401 assays of cognition using touchscreens as described above offers a powerful tool for future 402 investigations of the cognitive and behavioural predictors of $A B A$. 


\section{Acknowledgements}

404 The authors acknowledge the use of the facilities at Monash FlowCore and financial support from a

405 Research Training Program (RTP) stipend (LKM) and a Rebecca L Cooper Medical Research

406 Foundation Project Grant (CJF). Figures were created with resources from Biorender.com.

\section{Conflict of interest}

408 The authors report no competing financial disclosures or potential conflicts of interest. 


\section{References}

410

411

412

413

414

415

416

417

418

419

420

421

422

423

424

425

426

427

428

429

430

431

432

433

434

435

436

437

438

439

440

441

442

443

444

445

446

447

448

449

450

451

452

453

1. Arcelus, J., Mitchell, A.J., Wales, J. and Nielsen, S., Mortality rates in patients with anorexia nervosa and other eating disorders. A meta-analysis of 36 studies. Arch Gen Psychiatry, 2011. 68(7): p. 724-31.

2. Zipfel, S., Löwe, B., Reas, D.L., Deter, H.C. and Herzog, W., Long-term prognosis in anorexia nervosa: lessons from a 21-year follow-up study. Lancet, 2000. 355(9205): p. 7212.

3. Von Holle, A., Pinheiro, A.P., Thornton, L.M., Klump, K.L., Berrettini, W.H., Brandt, H., et al., Temporal patterns of recovery across eating disorder subtypes. Aust $\mathrm{N} Z \mathrm{Z}$ Psychiatry, 2008. 42(2): p. 108-17.

4. Le Grange, D. and Loeb, K.L., Early identification and treatment of eating disorders: prodrome to syndrome. Early Interv Psychiatry, 2007. 1(1): p. 27-39.

5. Mischoulon, D., Eddy, K.T., Keshaviah, A., Dinescu, D., Ross, S.L., Kass, A.E., et al., Depression and eating disorders: Treatment and course. J Affect Disord, 2010. 130(3): p. 470-477.

6. Thornton, L.M., Dellava, J.E., Root, T.L., Lichtenstein, P. and Bulik, C.M., Anorexia nervosa and generalized anxiety disorder: Further explorations of the relation between anxiety and body mass index. J Anxiety Disord, 2011. 25(5): p. 727-730.

7. Zerwas, S., Lund, B.C., Von Holle, A., Thornton, L.M., Berrettini, W.H., Brandt, H., et al., Factors associated with recovery from anorexia nervosa. J Psychiatr Res, 2013. 47(7): p. 972-979.

8. Keski-Rahkonen, A., Raevuori, A., Bulik, C.M., Hoek, H.W., Rissanen, A. and Kaprio, J., Factors Associated with Recovery from Anorexia Nervosa: A Population-Based Study. Int J Eat Disord, 2014. 47(2): p. 117-123.

9. Wild, B., Friederich, H.-C., Zipfel, S., Resmark, G., Giel, K., Teufel, M., et al., Predictors of outcomes in outpatients with anorexia nervosa - Results from the ANTOP study. Psychiatry Research, 2016. 244: p. 45-50.

10. Kaye, W.H., Bulik, C.M., Thornton, L., Barbarich, N. and Masters, K., Comorbidity of Anxiety Disorders With Anorexia and Bulimia Nervosa. Am J Psychiatry, 2004. 161(12): p. 2215-2221.

11. Ivarsson, T., Råstam, M., Wentz, E., Gillberg, I.C. and Gillberg, C., Depressive disorders in teenage-onset anorexia nervosa: A controlled longitudinal, partly community-based study. Compr Psychiatry, 2000. 41(5): p. 398-403.

12. Godart, N.T., Flament, M.F., Lecrubier, Y. and Jeammet, P., Anxiety disorders in anorexia nervosa and bulimia nervosa: co-morbidity and chronology of appearance. Eur Psychiatry, 2000. 15(1): p. 38-45.

13. Godart, N., Radon, L., Curt, F., Duclos, J., Perdereau, F., Lang, F., et al., Mood disorders in eating disorder patients: Prevalence and chronology of ONSET. J Affect Disord, 2015. 185: p. $115-122$.

14. Godart, N.T., Perdereau, F., Rein, Z., Berthoz, S., Wallier, J., Jeammet, P., et al., Comorbidity studies of eating disorders and mood disorders. Critical review of the literature. Journal of Affective Disorders, 2007. 97(1): p. 37-49.

15. Meier, S.M., Bulik, C.M., Thornton, L.M., Mattheisen, M., Mortensen, P.B. and Petersen, L., Diagnosed Anxiety Disorders and the Risk of Subsequent Anorexia Nervosa: A Danish Population Register Study. Eur Eat Disord Rev, 2015. 23(6): p. 524-530. 
454

455

456

457

458

459

460

461

462

463

464

465

466

467

468

469

470

471

472

473

474

475

476

477

478

479

480

481

482

483

484

485

486

487

488

489

490

491

492

493

494

495

496

497

498

499

500

16. Degortes, D., Zanetti, T., Tenconi, E., Santonastaso, P. and Favaro, A., Childhood Obsessive-compulsive Traits in Anorexia Nervosa Patients, Their Unaffected Sisters and Healthy Controls: A Retrospective Study. Eur Eat Disord Rev, 2014. 22(4): p. 237-242.

17. Anderluh, M.B., Tchanturia, K., Rabe-Hesketh, S. and Treasure, J., Childhood ObsessiveCompulsive Personality Traits in Adult Women With Eating Disorders: Defining a Broader Eating Disorder Phenotype. Am J Psychiatry, 2003. 160(2): p. 242-247.

18. Bailer, U.F., Frank, G.K., Price, J.C., Meltzer, C.C., Becker, C., Mathis, C.A., et al., Interaction between serotonin transporter and dopamine D2/D3 receptor radioligand measures is associated with harm avoidant symptoms in anorexia and bulimia nervosa. Psychiatry Research: Neuroimaging, 2013. 211(2): p. 160-168.

19. Strober, M., Lampert, C., Morrell, W., Burroughs, J. and Jacobs, C., A controlled family study of anorexia nervosa: Evidence of familial aggregation and lack of shared transmission with affective disorders. The International journal of eating disorders, 1990. 9(3): p. 239253.

20. Strober, M., Freeman, R., Lampert, C. and Diamond, J., The association of anxiety disorders and obsessive compulsive personality disorder with anorexia nervosa: Evidence from a family study with discussion of nosological and neurodevelopmental implications. Int J Eat Disord, 2007. 40(S3): p. S46-S51.

21. Bellodi, L., Cavallini, M.C., Bertelli, S., Chiapparino, D., Riboldi, C. and Smeraldi, E., Morbidity Risk for Obsessive-Compulsive Spectrum Disorders in First-Degree Relatives of Patients With Eating Disorders. Am J Psychiatry, 2001. 158(4): p. 563-569.

22. Dellava, J.E., Kendler, K.S. and Neale, M.C., Generalized anxiety disorder and anorexia nervosa: evidence of shared genetic variation. Depress Anxiety, 2011. 28(8): p. 728-733.

23. Wade, T.D., Bulik, C.M., Neale, M. and Kendler, K.S., Anorexia nervosa and major depression: shared genetic and environmental risk factors. The American journal of psychiatry., 2000. 157(3): p. 469.

24. Yilmaz, Z., Halvorsen, M., Bryois, J., Yu, D., Thornton, L.M., Zerwas, S., et al., Examination of the shared genetic basis of anorexia nervosa and obsessive-compulsive disorder. Mol Psychiatry, 2020. 25(9): p. 2036-2046.

25. Russo , S.J. and Nestler , E.J., The brain reward circuitry in mood disorders. Nature Reviews Neuroscience, 2013. 14(9): p. 609.

26. Gonçalves, Ó.F., Carvalho, S., Leite, J., Fernandes-Gonçalves, A., Carracedo, A. and Sampaio, A., Cognitive and emotional impairments in obsessive-compulsive disorder: Evidence from functional brain alterations. Porto Biomedical Journal, 2016. 1(3): p. 92-105.

27. Godier, L.R. and Park, R.J., Compulsivity in anorexia nervosa: a transdiagnostic concept. Frontiers in psychology, 2014. 5: p. 778.

28. Zerwas, S., Larsen, J.T., Petersen, L., Thornton, L.M., Quaranta, M., Koch, S.V., et al., Eating Disorders, Autoimmune, and Autoinflammatory Disease. Pediatrics, 2017. 140(6).

29. Duncan, L., Yilmaz, Z., Gaspar, H., Walters, R., Goldstein, J., Anttila, V., et al., Significant Locus and Metabolic Genetic Correlations Revealed in Genome-Wide Association Study of Anorexia Nervosa. Am J Psychiatry, 2017. 174(9): p. 850-858.

30. Pisetsky, D.S., Trace, S.E., Brownley, K.A., Hamer, R.M., Zucker, N.L., Roux-Lombard, P., et al., The expression of cytokines and chemokines in the blood of patients with severe weight loss from anorexia nervosa: an exploratory study. Cytokine, 2014. 69(1): p. 110-5.

31. Dalton, B., Bartholdy, S., Robinson, L., Solmi, M., Ibrahim, M.A.A., Breen, G., et al., A meta-analysis of cytokine concentrations in eating disorders. J Psychiatr Res, 2018. 103: p. 252-264. 
501

502

503

504

505

506

507

508

509

510

511

512

513

514

515

516

517

518

519

520

521

522

523

524

525

526

527

528

529

530

531

532

533

534

535

536

537

538

539

540

541

542

543

544

545

546

547

548

32. Wong, S. and Pinkney, J., Role of cytokines in regulating feeding behaviour. Curr Drug Targets, 2004. 5(3): p. 251-63.

33. Solmi, M., Veronese, N., Favaro, A., Santonastaso, P., Manzato, E., Sergi, G., et al., Inflammatory cytokines and anorexia nervosa: A meta-analysis of cross-sectional and longitudinal studies. Psychoneuroendocrinology, 2015. 51: p. 237-52.

34. Nilsson, I.A.K., Millischer, V., Göteson, A., Hübel, C., Thornton, L.M., Bulik, C.M., et al., Aberrant inflammatory profile in acute but not recovered anorexia nervosa. Brain Behav Immun, 2020. 88: p. 718-724.

35. Herpertz-Dahlmann, B., Seitz, J. and Baines, J., Food matters: how the microbiome and gut-brain interaction might impact the development and course of anorexia nervosa. Eur Child Adolesc Psychiatry, 2017. 26(9): p. 1031-1041.

36. Karagüzel, E., Arslan, F.C., Uysal, E.K., Demir, S., Aykut, D.S., Tat, M., et al., Blood levels of interleukin-1 beta, interleukin-6 and tumor necrosis factor-alpha and cognitive functions in patients with obsessive compulsive disorder. Compr Psychiatry, 2019. 89: p. 61-66.

37. Costello, H., Gould, R.L., Abrol, E. and Howard, R., Systematic review and meta-analysis of the association between peripheral inflammatory cytokines and generalised anxiety disorder. BMJ Open, 2019. 9(7): p. e027925.

38. Gutierrez, E., A rat in the labyrinth of anorexia nervosa: Contributions of the activity-based anorexia rodent model to the understanding of anorexia nervosa. 2013: Hoboken. p. 289301.

39. Schalla, M.A. and Stengel, A., Activity Based Anorexia as an Animal Model for Anorexia Nervosa-A Systematic Review. Front Nutr, 2019. 6: p. 69-69.

40. Scharner, S. and Stengel, A., Animal Models for Anorexia Nervosa-A Systematic Review. Front Hum Neurosci, 2021. 14: p. 596381-596381.

41. Milton, L.K., Oldfield, B.J. and Foldi, C.J., Evaluating anhedonia in the activity-based anorexia $(A B A)$ rat model. Physiol Behav, 2018. 194: p. 324-332.

42. Hurley, M.M., Murlanova, K., Macias, L.K., Sabir, A.I., O'Brien, S.C., Bhasin, H., et al., Activity-based anorexia disrupts systemic oxidative state and induces cortical mitochondrial fission in adolescent female rats. Int J Eat Disord, 2021. 54(4): p. 639-645.

43. Beeler, J.A., Mourra, D., Zanca, R.M., Kalmbach, A., Gellman, C., Klein, B.Y., et al., Vulnerable and Resilient Phenotypes in a Mouse Model of Anorexia Nervosa. Biol Psychiatry, 2020.

44. Kinzig, K.P. and Hargrave, S.L., Adolescent activity-based anorexia increases anxiety-like behavior in adulthood. Physiol Behav, 2010. 101(2): p. 269-276.

45. Chen, Y.-W., Surgent, O., Rana, B.S., Lee, F. and Aoki, C., Variant BDNF-Val66Met Polymorphism is Associated with Layer-Specific Alterations in GABAergic Innervation of Pyramidal Neurons, Elevated Anxiety and Reduced Vulnerability of Adolescent Male Mice to Activity-Based Anorexia. Cereb Cortex, 2017. 27(8): p. 3980-3993.

46. Chen, Y.W., Sherpa, A.D. and Aoki, C., Single injection of ketamine during mid-adolescence promotes long-lasting resilience to activity-based anorexia of female mice by increasing food intake and attenuating hyperactivity as well as anxiety-like behavior. Int J Eat Disord, 2018. 51(8): p. 1020-1025.

47. Wable, G.S., Min, J.-Y., Chen, Y.-W. and Aoki, C., Anxiety is correlated with running in adolescent female mice undergoing activity-based anorexia. Behavioral Neuroscience, 2015. 129(2): p. 170.

48. Belmonte, L., Achamrah, N., Nobis, S., Guérin, C., Riou, G., Bôle-Feysot, C., et al., A role for intestinal TLR4-driven inflammatory response during activity-based anorexia. Sci Rep, 2016. 6: p. 35813. 
549

550

551

552

553

554

555

556

557

558

559

560

561

562

563

564

565

566

567

568

569

570

571

572

573

574

575

576

577

578

579

580

581

582

583

584

585

586

587

588

589

590

591

592

593

594

595

596

49. Fasano, A., Intestinal permeability and its regulation by zonulin: diagnostic and therapeutic implications. Clin Gastroenterol Hepatol, 2012. 10(10): p. 1096-100.

50. Cora, M.C., Kooistra, L. and Travlos, G., Vaginal Cytology of the Laboratory Rat and Mouse: Review and Criteria for the Staging of the Estrous Cycle Using Stained Vaginal Smears. Toxicologic pathology, 2015. 43(6): p. 776-793.

51. File, S.E., Lippa, A.S., Beer, B. and Lippa, M.T., Animal Tests of Anxiety. Curr Protoc Neurosci, 2004. 26(1): p. 8.3.1-8.3.22.

52. Gould, T.D., Dao, D.T. and Kovacsics, C.E., The open field test, in Neuromethods. 2009. p. 1-20.

53. Prut, L. and Belzung, C., The open field as a paradigm to measure the effects of drugs on anxiety-like behaviors: a review: Animal models of anxiety disorders. European journal of pharmacology, 2003. 463(1-3): p. 3-33.

54. Thomas, A., Burant, A., Bui, N., Graham, D., Yuva-Paylor, L. and Paylor, R., Marble burying reflects a repetitive and perseverative behavior more than novelty-induced anxiety. Psychopharmacology, 2009. 204(2): p. 361-373.

55. Albelda, N. and Joel, D., Animal models of obsessive-compulsive disorder: Exploring pharmacology and neural substrates. Neurosci Biobehav Rev, 2012. 36(1): p. 47-63.

56. Lazic, S.E., Analytical strategies for the marble burying test: avoiding impossible predictions and invalid p-values. BMC Res Notes, 2015. 8(1): p. 141-141.

57. Dellava, J.E., Thornton, L.M., Hamer, R.M., Strober, M., Plotnicov, K., Klump, K.L., et al., Childhood anxiety associated with low BMI in women with anorexia nervosa. Behav Res Ther, 2010. 48(1): p. 60-7.

58. Rodgers, R.J., Animal models of 'anxiety': where next? Behavioural Pharmacology, 1997. 8(6).

59. Nosek, K., Dennis, K., Andrus, B.M., Ahmadiyeh, N., Baum, A.E., Woods, L.C.S., et al., Context and strain-dependent behavioral response to stress. Behavioral and Brain Functions, 2008. 4(1): p. 23.

60. Leonardo Rico, J., Hurtado-Parrado, C., Vásquez-Sepúlveda, J., Fonseca, J. and Cardona, Á., Time in the central area of the elevated plus-maze correlates with impulsivity-related measures during an operant task. Universitas Psychologica, 2016. 15: p. 1-9.

61. Tenconi, E., Degortes, D., Clementi, M., Collantoni, E., Pinato, C., Forzan, M., et al., Clinical and genetic correlates of decision making in anorexia nervosa. Journal of clinical and experimental neuropsychology, 2016. 38(3): p. 327-37.

62. Steward, T., Mestre-Bach, G., Agüera, Z., Granero, R., Martín-Romera, V., Sánchez, I., et al., Enduring Changes in Decision Making in Patients with Full Remission from Anorexia Nervosa. Eur Eat Disord Rev, 2016. 24(6): p. 523-527.

63. Sato, Y., Saito, N., Utsumi, A., Aizawa, E., Shoji, T., Izumiyama, M., et al., Neural basis of impaired cognitive flexibility in patients with anorexia nervosa. PloS one, 2013. 8(5): p. e61108.

64. Tchanturia, K., Davies, H., Roberts, M., Harrison, A., Nakazato, M., Schmidt, U., et al., Poor cognitive flexibility in eating disorders: examining the evidence using the Wisconsin Card Sorting Task. PloS one, 2012. 7(1): p. e28331.

65. Allen, P.J., Jimerson, D.C., Kanarek, R.B. and Kocsis, B., Impaired reversal learning in an animal model of anorexia nervosa. Physiol Behav, 2017. 179: p. 313-318.

66. Milton, L.K., Mirabella, P.N., Greaves, E., Spanswick, D.C., van den Buuse, M., Oldfield, B.J., et al., Suppression of Corticostriatal Circuit Activity Improves Cognitive Flexibility and Prevents Body Weight Loss in Activity-Based Anorexia in Rats. Biol Psychiatry, 2020. S0006-3223(20)31711-X. 
597

598

599

600

601

602

603

604

605

606

607

608

609

610

611

612

613

614

615

616

617

618

619

620

621

622

623

624

625

626

627

628

629

630

631

632

633

634

635

636

637

638

639

640

67. Romer, A.L. and Pizzagalli, D.A., Is executive dysfunction a risk marker or consequence of psychopathology? A test of executive function as a prospective predictor and outcome of general psychopathology in the adolescent brain cognitive development study ${ }^{\circledR}$. Dev Cogn Neurosci, 2021. 51: p. 100994.

68. Hurley, M.M., Collica, S.C., Aston, S.A., Wiles, L.J., Weiner, R.C., Biswas, A., et al., Adolescent female rats prone to the activity based anorexia (ABA) paradigm have altered hedonic responses and cortical astrocyte density compared to resistant animals. Appetite, 2021. 168: p. 105666.

69. Carrera, O., Gutiérrez, E. and Boakes, R.A., Early handling reduces vulnerability of rats to activity-based anorexia. Dev Psychobiol, 2006. 48(7): p. 520-7.

70. Novak, C.M., Burghardt, P.R. and Levine, J.A., The use of a running wheel to measure activity in rodents: Relationship to energy balance, general activity, and reward. Neurosci Biobehav Rev, 2012. 36(3): p. 1001-1014.

71. Miletta, M.C., Iyilikci, O., Shanabrough, M., Šestan-Peša, M., Cammisa, A., Zeiss, C.J., et al., AgRP neurons control compulsive exercise and survival in an activity-based anorexia model. Nat Metab, 2020. 2(11): p. 1204-1211.

72. de Brouwer, G., Fick, A., Harvey, B.H. and Wolmarans, D.W., A critical inquiry into marbleburying as a preclinical screening paradigm of relevance for anxiety and obsessivecompulsive disorder: Mapping the way forward. Cognitive, Affective, \& Behavioral Neuroscience, 2019. 19(1): p. 1-39.

73. Rosa Neto, J.C., Lira, F.S., Oyama, L.M., Zanchi, N.E., Yamashita, A.S., Batista, M.L., Jr., et al., Exhaustive exercise causes an anti-inflammatory effect in skeletal muscle and a proinflammatory effect in adipose tissue in rats. Eur J Appl Physiol, 2009. 106(5): p. 697-704.

74. Gomez-Merino, D., Drogou, C., Guezennec, C.Y. and Chennaoui, M., Effects of chronic exercise on cytokine production in white adipose tissue and skeletal muscle of rats. Cytokine, 2007. 40(1): p. 23-9.

75. Lira, F.S., Koyama, C.H., Yamashita, A.S., Rosa, J.C., Zanchi, N.E., Batista, M.L., Jr., et al., Chronic exercise decreases cytokine production in healthy rat skeletal muscle. Cell Biochem Funct, 2009. 27(7): p. 458-61.

76. Lin, S., Jin, S., Zhou, F., Hu, Y. and Zhang, M., Effects of endurance exercise on serum inflammatory cytokine level and kidney structure in a rat diabetes model. Exp Ther Med, 2021. 22(4): p. 1125.

77. Okita, N., Hayashida, Y., Kojima, Y., Fukushima, M., Yuguchi, K., Mikami, K., et al., Differential responses of white adipose tissue and brown adipose tissue to caloric restriction in rats. Mechanisms of Ageing and Development, 2012. 133(5): p. 255-266.

78. Dogan, S., Ray, A. and Cleary, M.P., The influence of different calorie restriction protocols on serum pro-inflammatory cytokines, adipokines and IGF-I levels in female C57BL6 mice: short term and long term diet effects. Meta Gene, 2017. 12: p. 22-32.

79. Zocher, S., Schilling, S., Grzyb, A.N., Adusumilli, V.S., Bogado Lopes, J., Günther, S., et al., Early-life environmental enrichment generates persistent individualized behavior in mice. Sci Adv, 2020. 6(35): p. eabb1478.

80. Caglayan, A., Stumpenhorst, K. and Winter, Y., Learning Set Formation and Reversal Learning in Mice During High-Throughput Home-Cage-Based Olfactory Discrimination. Front Behav Neurosci, 2021. 15: p. 684936. 


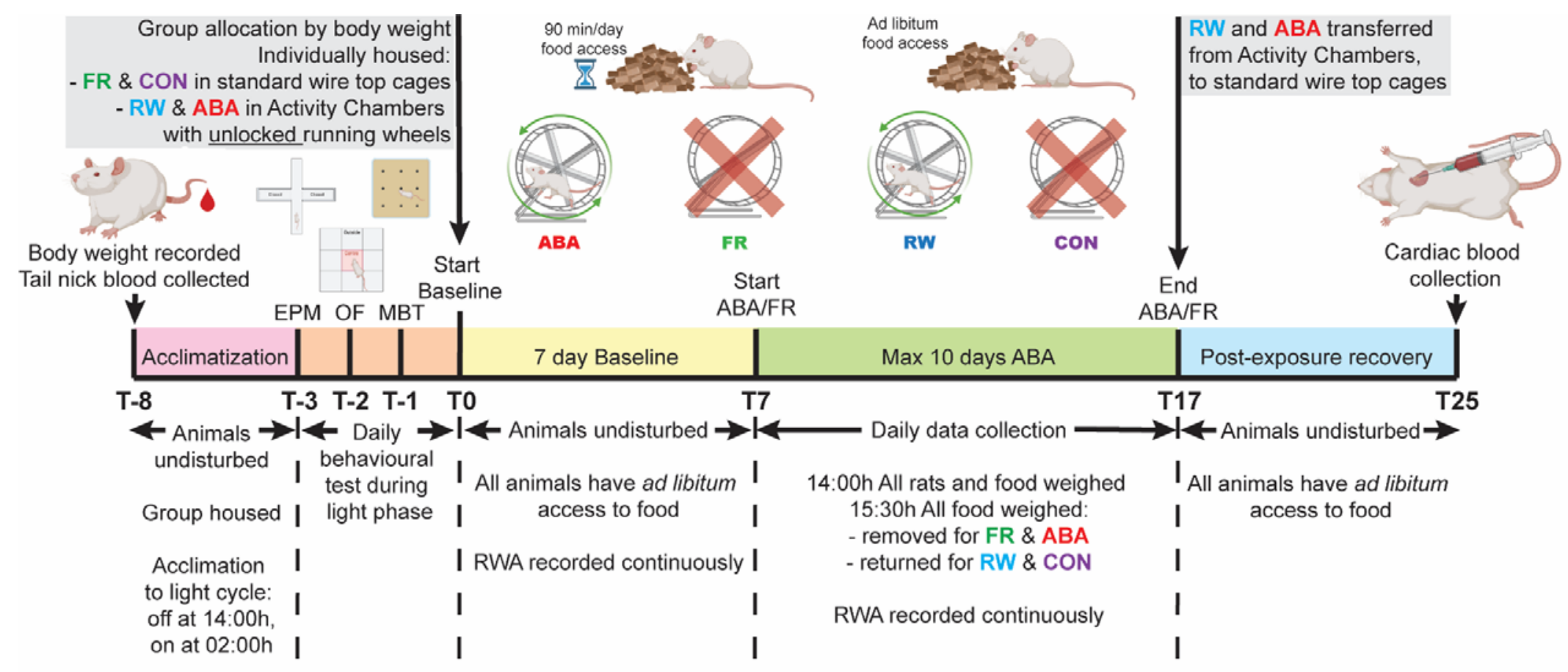

Figure 1 Experimental timeline. Pre-exposure blood samples were taken 8 days prior to the baseline testing period and rats were acclimated to the light cycle before maze-based behavioural tests on separate and consecutive days. The baseline testing period involved ad libitum food access and running activity was recorded for groups with access to wheels. Exposure to ABA conditions lasted for a maximum of 10 days, and all animals recovered with ad libitum access to food and no running wheels for 7 days post-exposure, followed by terminal blood collection. 
$\mathbf{A}$
$90 \mathrm{~min} / \mathrm{day}$ food access

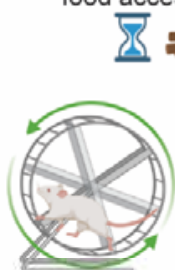

ABA

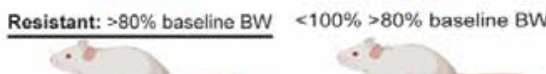

sceptible: $<80 \%$ baseline $8 \mathrm{WW}$

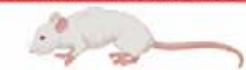

D

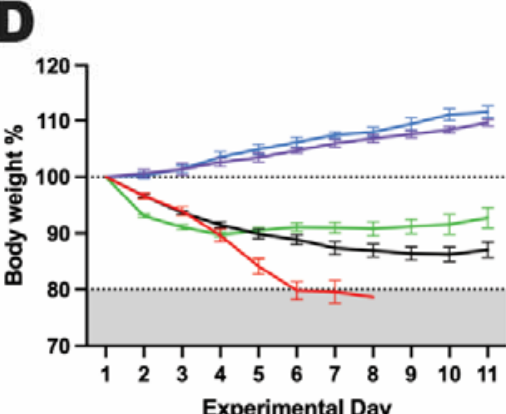

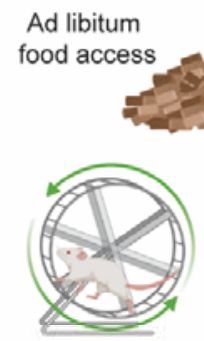

RW . b

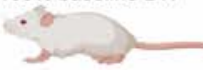

$>100 \%$ baseline BW

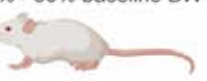

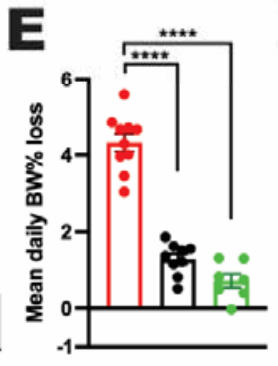

F

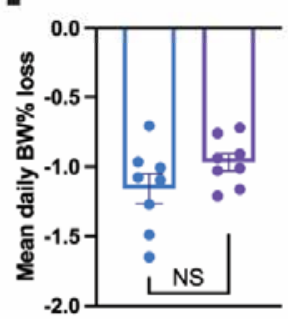

B

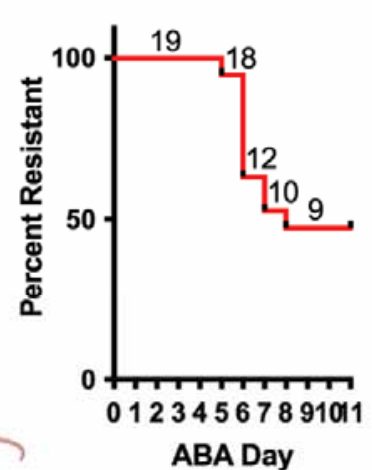

G

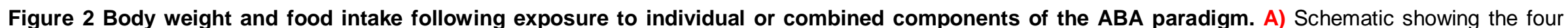

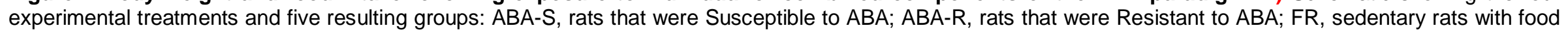

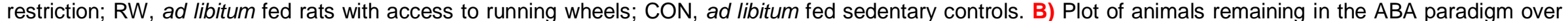

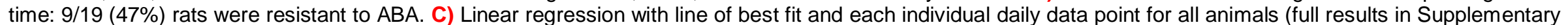

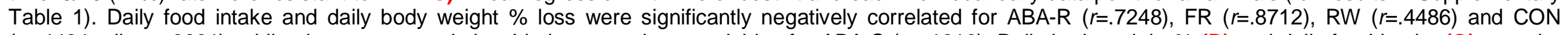

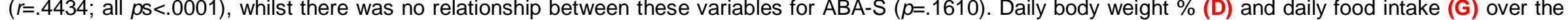

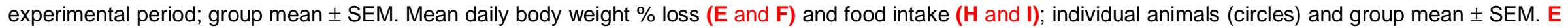

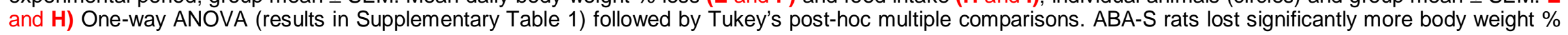

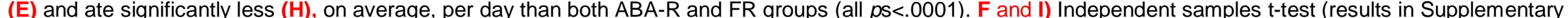

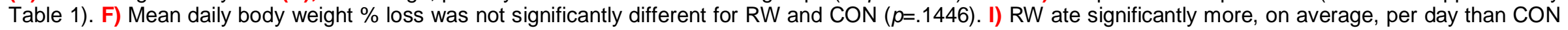
$(p=.0003)$. NS = not significant $p>.05,{ }^{* * *} p<.001,{ }^{* * *} p<.0001$. 
A

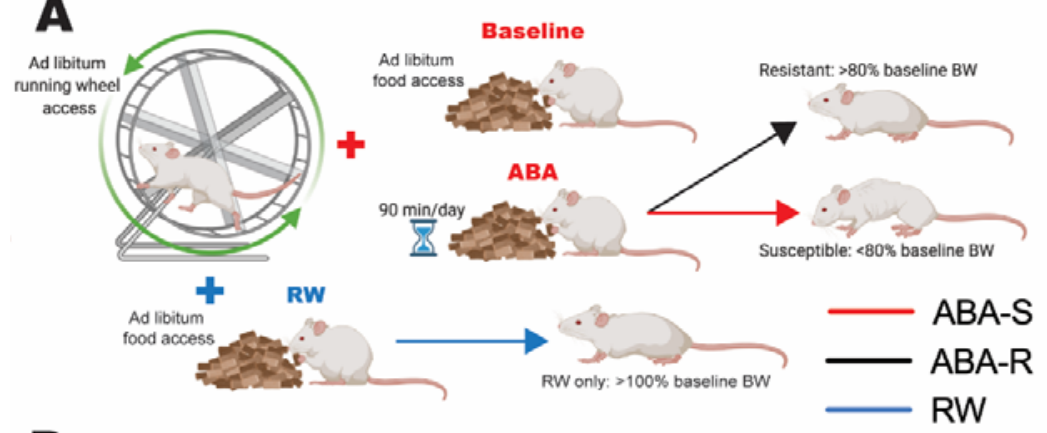

D

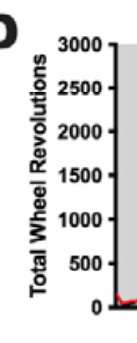

$\mathbf{F}$

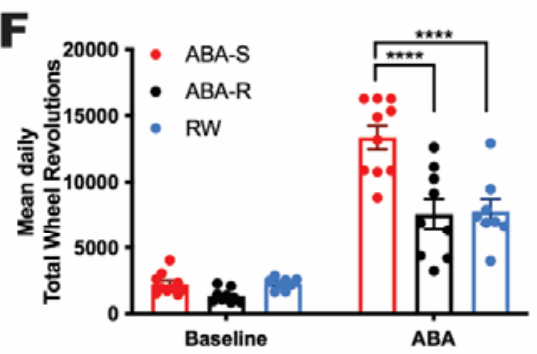

J

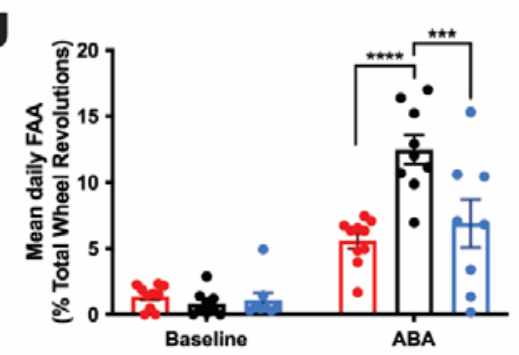

B
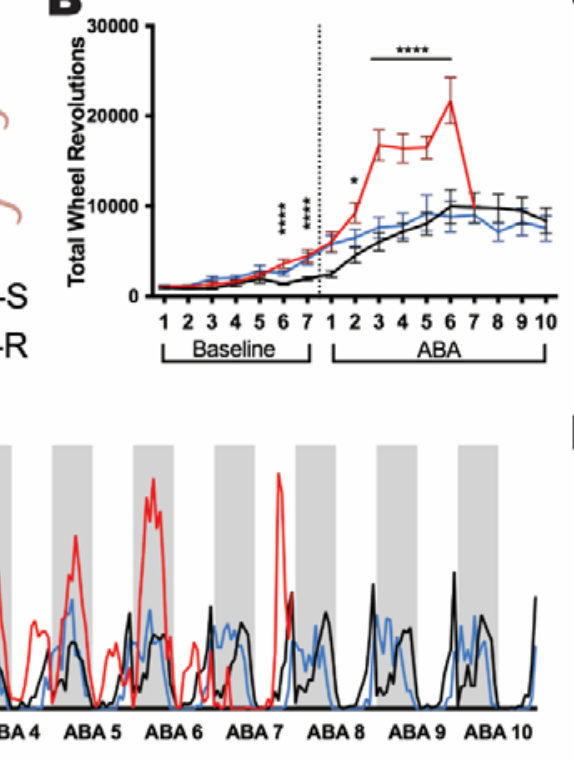

G

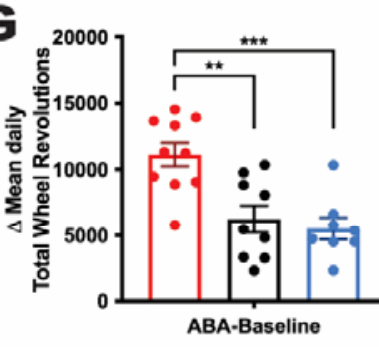

K

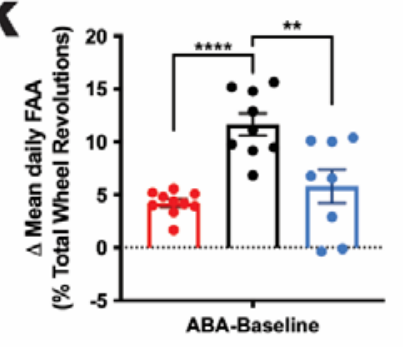

C

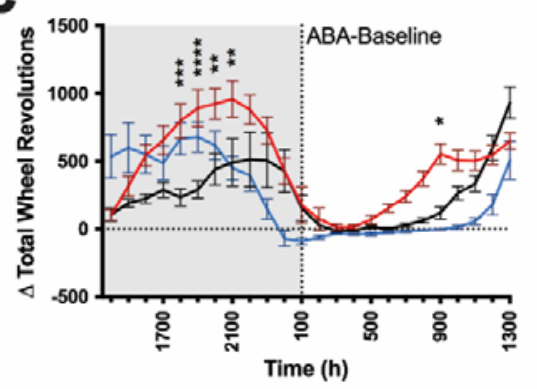

E。
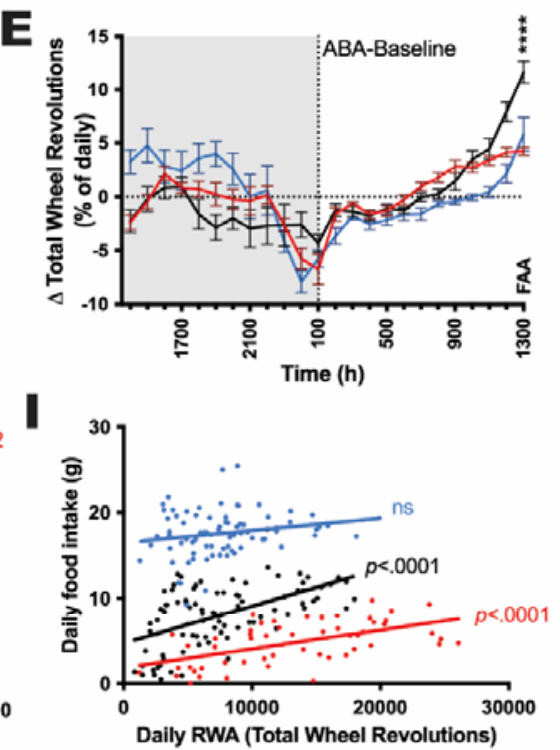

M
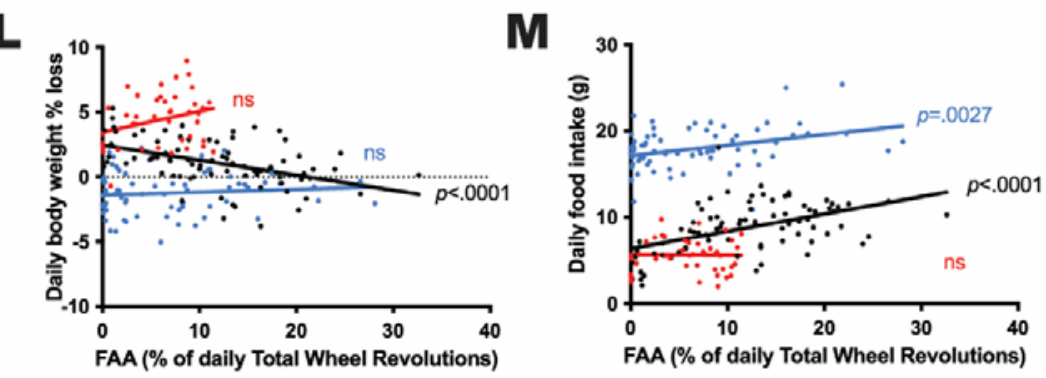
Figure 3 RWA for rats with wheel access either maintained on ad libitum feeding (RW) or combined with food restriction (ABA). A) Schematic showing the three experimental groups: Susceptible to ABA (ABA-S), Resistant to ABA (ABA-R), and those with access to running wheels but maintained on ad libitum feeding (RW). Group mean \pm SEM of daily RWA across the entirety of the experiment (B), change in mean hourly RWA across the dark (grey) and light (white) phases from baseline to ABA periods as absolute wheel revolutions (C), and as a proportion of total daily RWA (E). D) Hourly RWA across dark (grey) and light (white) phases for the entirety of the experiment; group mean without error shown for clarity. B, C, E) Two-way RM ANOVA (results in Supplementary Table 2) followed by post-hoc multiple comparisons with a Bonferroni correction were performed only on the ABA groups, RW is graphed solely for visual comparison. B) Overall, ABA-S rats had significantly greater daily RWA than ABA-R rats across both the Baseline $(p=.0101)$ and $A B A(p<.0001)$ periods. Baseline: Future ABA-S rats ran significantly more on baseline days 6 and 7 than future ABA-R rats (both $p s<.0001)$. ABA: ABA-S rats had significantly greater RWA than ABA-R rats on ABA day 2 ( $p=.014)$ and days 3-6 (all $p s<.0001)$. C) ABA-S rats had a significantly greater increase in hourly RWA from baseline to ABA than ABA-R rats from $1800 \mathrm{~h}-2100 \mathrm{~h}$ (all $p S<.0063$ ), and $0900 \mathrm{~h}(p=.0156)$. E) ABA-R rats had a significantly greater increase in proportional mean hourly RWA from baseline to ABA than ABA-S rats in the penultimate hour before food access $(1300 \mathrm{~h} ; p<.0001)$. Mean daily RWA $(\mathrm{F})$ and proportional FAA (J) during baseline and ABA phases, and change in mean daily RWA (G) and proportional FAA (K) from baseline to ABA; individual animals (circles) and group mean \pm SEM. F and J) Two-way RM ANOVA (results in Supplementary Table 2) followed by post-hoc multiple comparisons, Tukey's for between groups or with a Bonferroni correction for between phase. G and K) One-way ANOVA (results in Supplementary Table 2) followed by Tukey's post-hoc multiple comparisons. H, I, L, M) Linear regression with line of best fit and each individual daily data point for all animals (full results in Supplementary Table 2). F) ABA-S rats ran significantly more, on average, per day in the ABA phase than both ABA-R and RW rats (both $p s<.0001)$. G) The magnitude of increase in mean daily RWA from baseline to ABA was significantly greater in ABA-S rats than both ABA-R ( $p=.0022)$ and $R W$ $(p=.0008)$ rats. H) Daily RWA and Daily body weight \% loss were significantly positively correlated for ABA-S $(r=.4970, p=.0002)$ and RW $(r=.3553, p=.0012)$ animals, with no relationship between these variables for ABA-R animals $(p=1476)$. I) Daily RWA and Daily food intake were significantly positively correlated for ABA-S ( $r=.5574, p<.0001)$ and ABA-R ( $r=.5508, p<.0001)$ animals, whilst there was no relationship between these variables for RW animals $(p=.0774)$. J) ABA-R rats had significantly greater FAA in the ABA phase than both ABA-S $(p<.0001)$ and RW $(p=.0002)$ rats. K) The magnitude of increase in mean daily FAA from baseline to ABA was significantly greater in ABA-R rats than both ABA-S $(p<.0001)$ and RW $(p=.0019)$ rats. L) Daily FAA and Daily body weight \% loss were significantly negatively correlated for ABA-R $(r=.4569, p<.0001)$ while there was no relationship between these variables for either ABA-S ( $p=.0666)$ or RW $(p=.4237)$ animals. M) Daily FAA and Daily food intake were significantly positively correlated for both ABA-R $(r=.4876, p<.0001)$ and $\mathrm{RW}(r=.3484, p=.0027)$ animals, but there was no relationship between these variables for ABA-S animals $(p=.9293) .{ }^{*} p<.05,{ }^{* *} p<.01,{ }^{* * *} p<.001,{ }^{* * *} p<.0001$. 


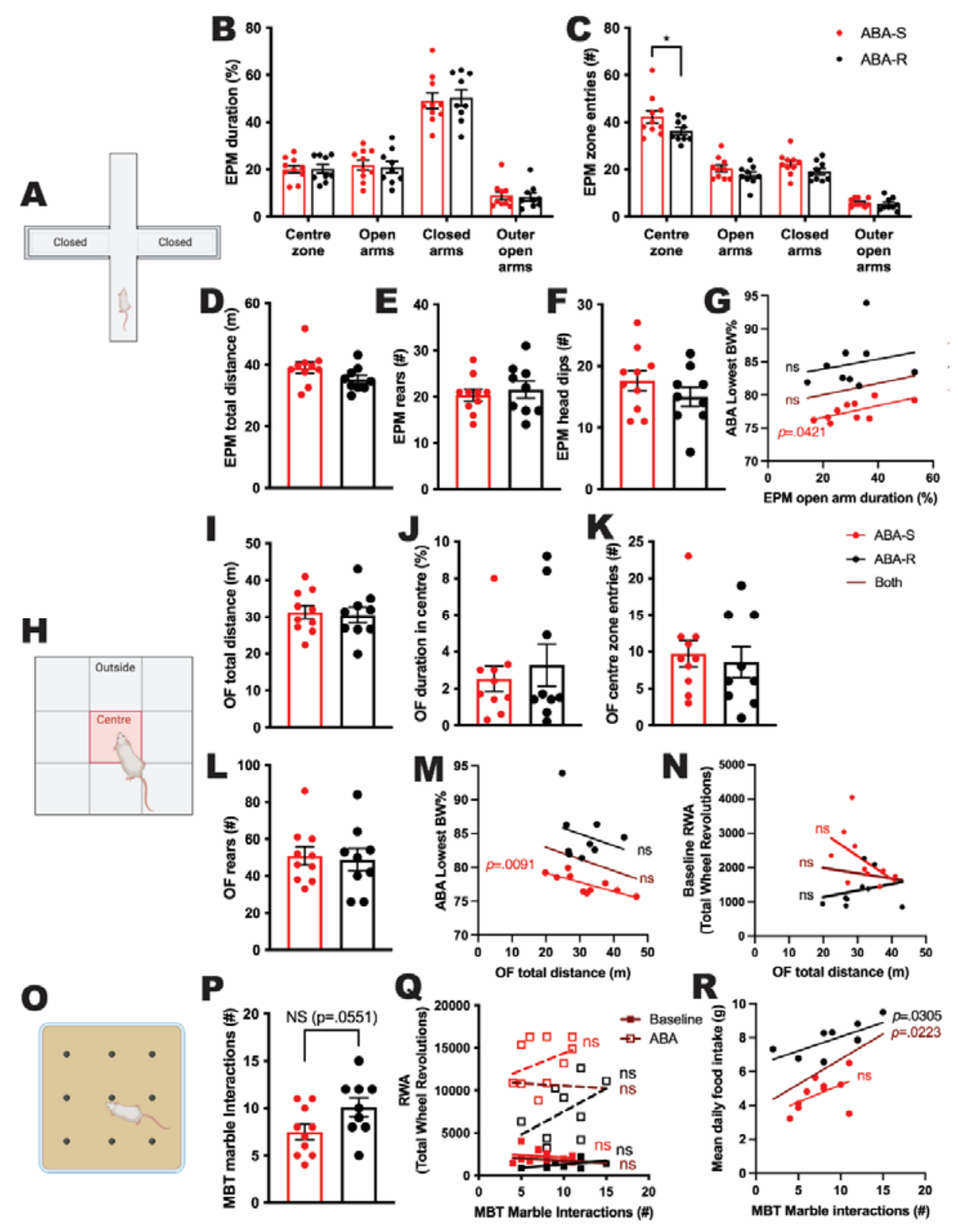

Figure 4 Pre-exposure behavioural test results and correlations with ABA outcomes. A) Schematic of the EPM; outer open arms are not shown but constituted the end 1/3 of each open arm. Bar graphs show group mean \pm SEM with individual animals (circles) for animals that went on to be Susceptible to ABA (ABAS) or Resistant to ABA (ABA-R). B and C) Two-way RM ANOVA (results in Supplementary Table 3). B) Duration (\%) spent in each of the EPM zones did not differ significantly between groups. C) Number of entries into each of the EPM zones. Future ABA-S rats made significantly more entries into the centre zone than future ABA-R rats $(p=.0355)$. D-F, I-L, P) Independent samples t-test (results in Supplementary Table 3 ). Neither the total distance (D), number of exploratory rears $(E)$, nor number of head dips (F) on the EPM differed between groups (all ps>.1215). G, M, N, Q, R) Linear regression with line of best fit and all individual animal data points; line of best fit for the combined ABA group is also shown (full results in Supplementary Table 3). G) The duration of time spent in the open arms of the EPM was significantly positively correlated for ABA-S rats $(r=.6495, p=.0421)$, whilst there was no relationship between these variables for ABA-R $(p=.5801)$ nor the combined ABA group $(p=.4248)$. H) Schematic of the OF. None of the OF outcome measures differentiated between rats that went on to be Susceptible or Resistant to ABA (all pS>.5724): I) Total distance; J) Centre duration (\%); K) Centre zone entries; L) Exploratory rears. M) OF total distance was significantly negatively correlated with subsequent lowest body weight $\%$ during ABA for ABA-S rats $(r=.7706, p=.0091)$, but not for ABA-R rats $(p=.4891)$ or the combined ABA group $(p=.2753)$. N) There was 
bioRxiv preprint doi: https://doi.org/10.1101/2021.10.25.465801; this version posted October 26, 2021. The copyright holder for this preprint (which was not certified by peer review) is the author/funder, who has granted bioRxiv a license to display the preprint in perpetuity. It is made available under aCC-BY-NC-ND 4.0 International license.

no relationship between OF total distance and mean daily Baseline RWA for any group (all ps>.1538). O) Schematic of the MBT. P) The number of interactions with marbles was not significantly different between groups $(p=.0551)$, was not significantly correlated with mean daily RWA during either Baseline or ABA for any group (Q; all $p s>$.1873), but was significantly positively correlated with 90-minute food intake during ABA (R) for ABA-R ( $r=.7147, p=.0305)$ and the combined ABA group ( $r=.5205, p=.0223)$, but not for ABA-S $(p=.1319)$. 


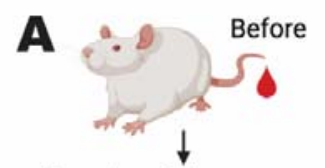

Experimental exposure

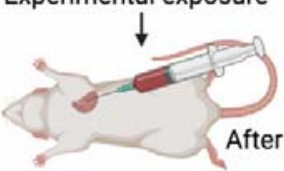

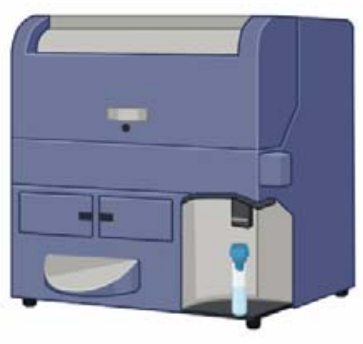

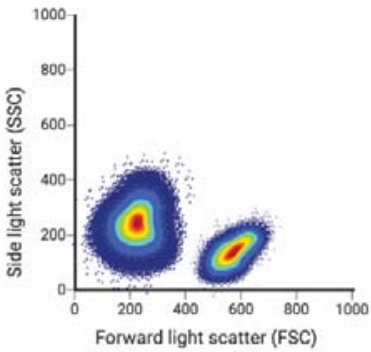

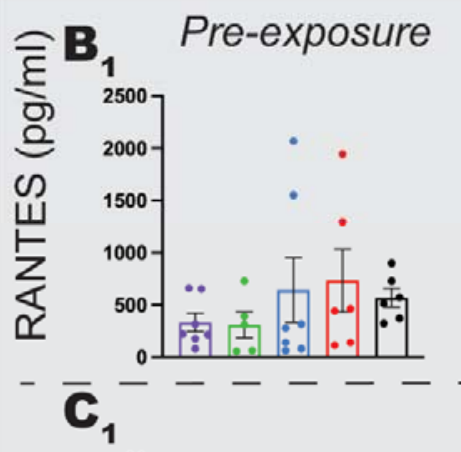

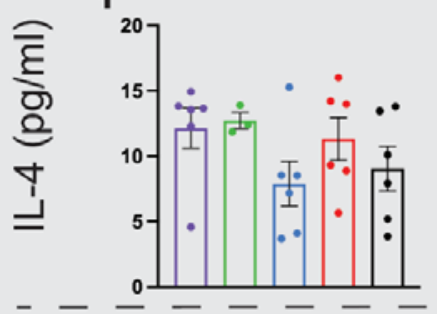

- $\overline{\mathbf{D}}$

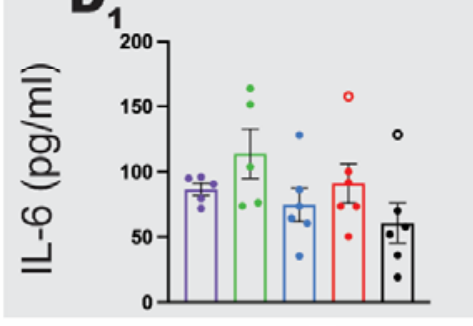

$\mathbf{E}$
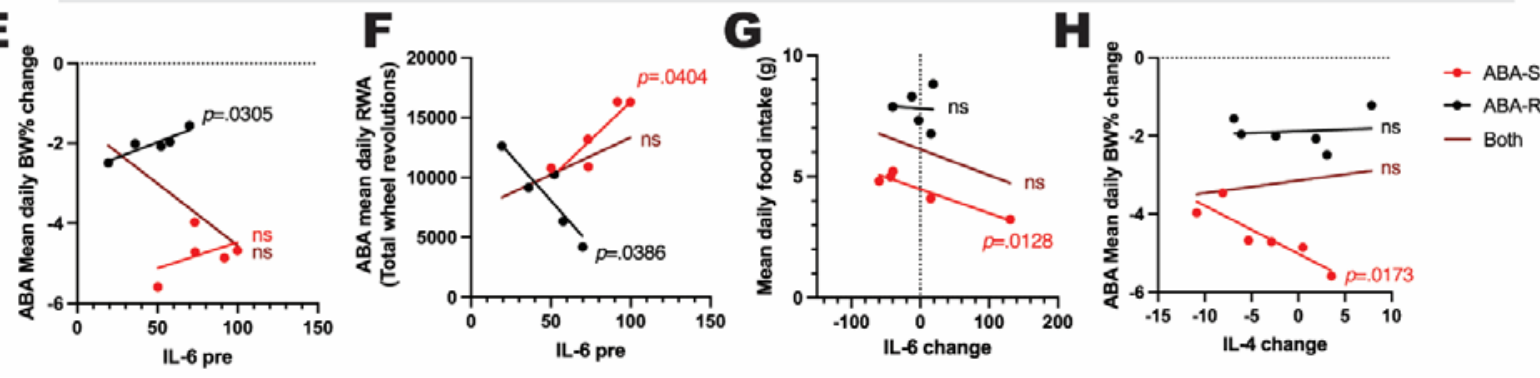

Figure 5 Concentration of proinflammatory cytokines before and after exposure to ABA conditions A) Schematic of blood collection and flow cytometry timeline. B-D) Plasma cytokine concentration $(\mathrm{pg} / \mathrm{mL})$ collected from rats before experimental exposure (1), at the cessation of the experiment (2) and the change from before to after (3) for RANTES (B), IL-4 (C), and IL-6 (D). Welch's One-way ANOVAs revealed no significant differences between groups for any cytokine (all $p s>.0581$; results in Supplementary Table 4). EG) Linear regression with line of best fit and all individual animal data points (full results in Supplementary Table 4); NOTE: two outlying IL-6 pre-exposure values (1 from each ABA subgroup; open circles in D), and the corresponding change values, were excluded from linear regression analyses leaving $n=5$ in each group. E) Pre-exposure levels of IL-6 were significantly positively correlated with mean daily body weight $\%$ change in ABA, such that higher pre-exposure IL-6 was associated with less weight loss, for ABA-R ( $r=.9128$, 
bioRxiv preprint doi: https://doi.org/10.1101/2021.10.25.465801; this version posted October 26, 2021. The copyright holder for this preprint (which was not certified by peer review) is the author/funder, who has granted bioRxiv a license to display the preprint in perpetuity. It is made available under aCC-BY-NC-ND 4.0 International license.

$p=.0305)$, but not ABA-S ( $p=.4627)$ nor the combined ABA group ( $p=.1396)$. F) Pre-exposure levels of IL-6 were significantly correlated with mean daily RWA during ABA for both ABA subgroups, although in opposite directions; positive for ABA-S ( $r=.8947, p=.0404)$ and negative for ABA-R $(r=.8978, p=.0386)$. Consequently, there was no significant relationship between these variables for the combined ABA group $(p=.2617)$. G) The change in IL-6 from pre- to post-exposure was significantly negatively correlated with mean 90-minute food intake during ABA for ABA-S ( $r=.9513, p=.0128)$, but not ABA-R $(p=.9219)$ nor the combined ABA group $(p=.3963)$. H) The change in IL-4 from pre- to post-exposure was significantly negatively correlated with mean daily body weight $\%$ change in ABA for ABA-S $(r=.8907, p=.0173)$, but not ABA-R $(p=.8211)$, nor the combined ABA group $(p=.7101)$. 
Supplementary Table 1 Statistical test details and results for data presented in Figure 2

\begin{tabular}{|c|c|c|c|}
\hline Figure & Statistical test & Main analysis result & Post-hoc multiple comparisons \\
\hline 2B & Linear regression & $\begin{array}{l}\text { ABA-S: } r=.1935, \mathrm{R}^{2}=.03816, p=.1610 \\
\text { ABA-R: } r=.7248, \mathrm{R}^{2}=.5254, \boldsymbol{p}<.0001 \\
\text { FR: } r=.8712, \mathrm{R}^{2}=.7509, \boldsymbol{p}<.0001 \\
\text { RW: } r=.4486, \mathrm{R}^{2}=.2012, \boldsymbol{p}<.0001 \\
\text { CON: } r=.4434, \mathrm{R}^{2}=.1966, \boldsymbol{p}<.0001\end{array}$ & \\
\hline 2E & One-way ANOVA & $F(2,23)=101.3, \boldsymbol{p}<.0001$ & $\begin{array}{l}\text { ABA-S }>\text { ABA-R, } \boldsymbol{p}<.0001 \\
\text { ABA-S }>\text { FR, } \boldsymbol{p}<.0001\end{array}$ \\
\hline 2F & Independent samples t-test & $t(14)=1.545, p=.1446$ & \\
\hline $2 \mathrm{H}$ & One-way ANOVA & $F(2,23)=27.29, \boldsymbol{p}<.0001$ & $\begin{array}{l}\text { ABA-S }<\text { ABA-R, } \boldsymbol{p}<.0001 \\
\text { ABA-S }<\text { FR, } \boldsymbol{p}<.0001\end{array}$ \\
\hline 2I & Independent samples t-test & $t(14)=4.846, p=.0003$ & \\
\hline
\end{tabular}


Supplementary Table 2 Statistical test details and results for data presented in Figure 3

\begin{tabular}{|c|c|c|c|}
\hline Figure & Statistical test & Main analysis result & Post-hoc multiple comparisons \\
\hline $3 B$ & $\begin{array}{l}\text { Baseline: Two-way RM ANOVA } \\
\text { ONLY INCLUDES ABA-S and ABA-R }\end{array}$ & $\begin{array}{l}\text { Time } F(6,102)=20.24, \boldsymbol{p}<.0001 \\
\text { Outcome } F(1,17)=8.378, \boldsymbol{p}=.0101 \\
\text { Interaction } F(6,102)=6.850, \boldsymbol{p}<.0001\end{array}$ & $\begin{array}{l}\text { Day 6: ABA-S > ABA-R } \boldsymbol{p}<.0001 \\
\text { Day 7: ABA-S }>\text { ABA-R } \boldsymbol{p}<.0001\end{array}$ \\
\hline 3B & $\begin{array}{l}\text { ABA: Mixed-model ANOVA (due to missing values from } \\
\text { ABA-S rats removed from the experiment over time) } \\
\text { ONLY INCLUDES ABA-S and ABA-R }\end{array}$ & $\begin{array}{l}\text { Time } F(9,109.996)=20.644, \boldsymbol{p}<.0001 \\
\text { Outcome } F(1,20.878)=24.739, \boldsymbol{p}<.0001 \\
\text { Interaction } F(6,110.337)=5.431, \boldsymbol{p}<.0001\end{array}$ & $\begin{array}{l}\text { Day 2: ABA-S }>A B A-R p=.014 \\
\text { Days 3-6: ABA-S }>\text { ABA-R all } p s<.0001\end{array}$ \\
\hline 3C & $\begin{array}{l}\text { Two-way RM ANOVA } \\
\text { ONLY INCLUDES ABA-S and ABA-R }\end{array}$ & $\begin{array}{l}\text { Time } F(23,391)=18.93, \boldsymbol{p}<.0001 \\
\text { Outcome } F(1,17)=13.06, \boldsymbol{p}=.0021 \\
\text { Interaction } F(23,391)=3.574, \boldsymbol{p}<.0001\end{array}$ & $\begin{array}{l}\text { 1800h: ABA-S > ABA-R } p=.0003 \\
\text { 1900h: ABA-S > ABA-R } p<.0001 \\
\text { 2000h: ABA-S > ABA-R } p=.0036 \\
\text { 2100h: ABA-S > ABA-R } p=.0063 \\
\text { 0900h: ABA-S > ABA-R } p=. \mathbf{0 1 5 6}\end{array}$ \\
\hline 3E & Two-way RM ANOVA & $\begin{array}{l}\text { Time } F(23,391)=22.33, \boldsymbol{p}<.0001 \\
\text { Outcome } F(1,17)=0.1744, p=.6814 \\
\text { Interaction } F(23,391)=3.433, \boldsymbol{p}<.0001\end{array}$ & 1300h: ABA-R > ABA-S $p<.0001$ \\
\hline 3F & Two-way RM ANOVA & $\begin{array}{l}\text { Phase } F(1,24)=204.3, p<.0001 \\
\text { Group } F(2,24)=11.66, p=.0003 \\
\text { Interaction } F(2,24)=11.33, p=.0003\end{array}$ & $\begin{array}{l}\text { Phase: all groups ABA > Baseline } \boldsymbol{p}<.0001 \\
\text { Group: ABA-S }>\text { ABA-R } p=.0004 ; A B A-S>R W p=.0038 \\
\text { ABA phase: ABA-S }>\text { ABA-R } p<.0001 ; A B A-S>R W \\
p<.0001\end{array}$ \\
\hline 3G & One-way ANOVA & $F(2,24)=11.33, p=.0003$ & $\begin{array}{l}\mathrm{ABA}-\mathrm{S}>\mathrm{ABA}-\mathrm{R} \boldsymbol{p}=.0022 \\
\mathrm{ABA}-\mathrm{S}>\mathrm{RW} \boldsymbol{p}=.0008\end{array}$ \\
\hline $3 \mathrm{H}$ & Linear regression & $\begin{array}{l}\text { ABA-S: } r=.4970, R^{2}=.2470, p=.0002 \\
\text { ABA-R: } r=.1539, R^{2}=.02367, p=.1476 \\
\text { RW: } r=.3553, R^{2}=.1263, p=.0012\end{array}$ & \\
\hline 3I & Linear regression & $\begin{array}{l}\text { ABA-S: } r=.5574, R^{2}=.3107, p<.0001 \\
\text { ABA-R: } r=.5508, R^{2}=.3034, p<.0001 \\
\text { RW: } r=.1986, R^{2}=.03945, p=.0774\end{array}$ & \\
\hline 3J & Two-way RM ANOVA & $\begin{array}{l}\text { Phase } F(1,24)=146.6, \boldsymbol{p}<.0001 \\
\text { Group } F(2,24)=5.870, \boldsymbol{p}=.0084 \\
\text { Interaction } F(2,24)=14.84), \boldsymbol{p}<.0001\end{array}$ & $\begin{array}{l}\text { Phase: ABA-S } p=.0007 ; \text { ABA-R } p<.0001 \text {; RW } p<.0001 \\
\text { Group: ABA-R }>\text { ABA-S } p=.0093 ; \text { ABA-R }>\text { RW } p=.0430 \\
\text { ABA phase: ABA-R }>\text { ABA-S } p<.0001 ; A B A-R>R W \\
p=.0002\end{array}$ \\
\hline 3K & One-way ANOVA & $F(2,24)=14.84, \boldsymbol{p}<.0001$ & $\begin{array}{l}\mathrm{ABA}-\mathrm{R}>\mathrm{ABA}-\mathrm{S} p<.0001 \\
\mathrm{ABA}-\mathrm{R}>\mathrm{RW} p=.0019\end{array}$ \\
\hline 3L & Linear regression & $\begin{array}{l}\text { ABA-S: } r=.2823, \mathrm{R}^{2}=.07969, p=.0666 \\
\text { ABA-R: } r=.4569, \mathrm{R}^{2}=.2088, p<.0001 \\
\mathrm{RW}: r=.0957, \mathrm{R}^{2}=.009167, p=.4237\end{array}$ & \\
\hline $3 M$ & Linear regression & $\begin{array}{l}\text { ABA-S: } r=.0139, \mathrm{R}^{2}=.0007975, p=.9293 \\
\text { ABA-R: } r=.4876, \mathrm{R}^{2}=.2378, p<.0001 \\
\text { RW: } r=.3484, \mathrm{R}^{2}=.1214, p=.0027\end{array}$ & \\
\hline
\end{tabular}


Supplementary Table 3 Statistical test details and results for data presented in Figure 4

\begin{tabular}{|c|c|c|c|}
\hline Figure & Statistical test & Main analysis result & Post-hoc multiple comparisons \\
\hline 4B & Two-way RM ANOVA & $\begin{array}{l}\text { Zone } F(3,68)=111.2, p<.0001 \\
\text { ABA outcome } F(1,68)=7.200 \mathrm{e}-006, p=.9979 \\
\text { Interaction } F(3,68)=0.08631, p=.9673\end{array}$ & \\
\hline 4C & Two-way RM ANOVA & $\begin{array}{l}\text { Zone } F(3,68)=155.5, p<.0001 \\
\text { ABA outcome } F(1,68)=8.307, p=.0053 \\
\text { Interaction } F(3,68)=0.9624, p=.4156\end{array}$ & Centre zone: ABA-S > ABA-R $p=. \mathbf{0 3 5 5}$ \\
\hline 4D & Independent samples t-test & $t(17)=1.630, p=.1215$ & \\
\hline 4E & Independent samples t-test & $t(17)=0.5274, p=.6048$ & \\
\hline 4F & Independent samples t-test & $t(17)=1.138, p=.2709$ & \\
\hline 4G & Linear regression & $\begin{array}{l}\text { ABA-S: } r=.6495, \mathrm{R}^{2}=.4219, p=.0421 \\
\text { ABA-R: } r=.2141, \mathrm{R}^{2}=.04586, p=.5801 \\
\text { Combined ABA: } r=.1946, \mathrm{R}^{2}=.03785, p=.4248\end{array}$ & \\
\hline 41 & Independent samples t-test & $t(17)=0.1753, p=.8629$ & \\
\hline 4J & Independent samples t-test & $t(17)=0.5757, p=.5724$ & \\
\hline $4 \mathrm{~K}$ & Independent samples t-test & $t(17)=0.4199, p=.6799$ & \\
\hline 4L & Independent samples t-test & $t(17)=0.2738, p=.7875$ & \\
\hline $4 \mathrm{M}$ & Linear regression & $\begin{array}{l}\text { ABA-S: } r=.7706, \mathrm{R}^{2}=.5938, p=.0091 \\
\text { ABA-R: } r=.2660, \mathrm{R}^{2}=.07075, p=.4891 \\
\text { Combined ABA: } r=.2637, \mathrm{R}^{2}=.06955, p=.2753\end{array}$ & \\
\hline $4 N$ & Linear regression & $\begin{array}{l}\text { ABA-S: } r=.4866, \mathrm{R}^{2}=.2368, p=.1538 \\
\text { ABA-R: } r=.2435, \mathrm{R}^{2}=.05931, p=.5278 \\
\text { Combined ABA: } r=.1152, \mathrm{R}^{2}=.01326, p=.6387\end{array}$ & \\
\hline 4P & Independent samples t-test & $t(17)=2.060, p=.0551$ & \\
\hline $4 Q$ & Linear regression & $\begin{array}{l}\frac{\text { Baseline }}{\text { ABA-S: } r=.1793, \mathrm{R}^{2}=.03216, p=.6201} \\
\text { ABA-R: } r=.4835, \mathrm{R}^{2}=.2338, p=.1873 \\
\text { Combined ABA: } r=.1954, \mathrm{R}^{2}=.03819, p=.4227 \\
\text { ABA } \\
\text { ABA-S: } r=.3607, \mathrm{R}^{2}=.1301, p=.3059 \\
\text { ABA-R: } r=.4800, \mathrm{R}^{2}=.2304, p=.1909 \\
\text { Combined ABA: } r=.0430, \mathrm{R}^{2}=.001853 \\
p=.8611\end{array}$ & \\
\hline 4R & Linear regression & $\begin{array}{l}\text { ABA-S: } r=.5102, R^{2}=.2603, p=.1319 \\
\text { ABA-R: } r=.7147, R^{2}=.5108, p=.0305 \\
\text { Combined ABA: } r=.5205, R^{2}=.2709, p=.0223\end{array}$ & \\
\hline
\end{tabular}


Supplementary Table 4 Statistical test details and results for data presented in Figure 5

\begin{tabular}{|c|c|c|c|}
\hline Figure & Statistical test & Main analysis result & Group size \\
\hline 5B1 & Welch's One-way RM ANOVA & $\mathrm{W}(4,12.27)=1.299, p=.3238$ & \multirow{3}{*}{$\begin{array}{l}\text { RANTES: ABA-S } n=6, \text { ABA-R } n=6, F R n=6, R W n=7, \text { CON } \\
n=7\end{array}$} \\
\hline 5B2 & Welch's One-way RM ANOVA & $\mathrm{W}(4,12.34)=0.8452, p=.5223$ & \\
\hline 5B3 & Welch's One-way RM ANOVA & $\mathrm{W}(4,12.54)=1.077, p=.4086$ & \\
\hline 5C1 & Welch's One-way RM ANOVA & $W(4,11)=, 2.172 p=.1394$ & \multirow[t]{3}{*}{ IL-4: ABA-S n=6, ANA-R n=6, FR n=3, RW n=6, CON n=6 } \\
\hline $5 C 2$ & Welch's One-way RM ANOVA & $\mathrm{W}(4,8.492)=0.7422, p=.5880$ & \\
\hline 5C3 & Welch's One-way RM ANOVA & $\mathrm{W}(4,10.69)=3.202, p=.0581$ & \\
\hline 5D1 & Welch's One-way RM ANOVA & $\mathrm{W}(4,10.57)=1.218, p=.3599$ & \multirow[t]{3}{*}{ IL-6: ABA-S n=6, ABA-R n=6, FR n=6, RW n=6, CON n=5 } \\
\hline $5 \mathrm{D2}$ & Welch's One-way RM ANOVA & $\mathrm{W}(4,10.63)=0.3845, p=.8152$ & \\
\hline 5D3 & Welch's One-way RM ANOVA & $\mathrm{W}(4,10.52)=1.132, p=.3929$ & \\
\hline $\mathbf{E}$ & Linear regression & $\begin{array}{l}\text { ABA-S: } r=.4362, \mathrm{R}^{2}=.1903, p=.4627 \\
\text { ABA-R: } r=.9128, \mathrm{R}^{2}=.8332, p=.0305 \\
\text { Combined ABA: } r=.5017, \mathrm{R}^{2}=.2517, p=.1396\end{array}$ & \multirow[t]{3}{*}{ IL-6: ABA-S n=5, ABA-R n=5, Combined ABA $n=10$} \\
\hline $\mathbf{F}$ & Linear regression & $\begin{array}{l}\text { ABA-S: } r=.8947, \mathrm{R}^{2}=.8004, p=.0404 \\
\text { ABA-R: } r=.8978, \mathrm{R}^{2}=.8061, p=.0386 \\
\text { Combined ABA: } r=.3927, \mathrm{R}^{2}=.1542, p=.2617\end{array}$ & \\
\hline $\mathbf{G}$ & Linear regression & $\begin{array}{l}\text { ABA-S: } r=.9513, \mathrm{R}^{2}=.9049, p=.0128 \\
\text { ABA-R: } r=.0614, \mathrm{R}^{2}=.003770, p=.9219 \\
\text { Combined ABA: } r=.3021, \mathrm{R}^{2}=.09124, p=.3963\end{array}$ & \\
\hline H & Linear regression & $\begin{array}{l}\text { ABA-S: } r=.8907, R^{2}=.7934, p=.0173 \\
\text { ABA-R: } r=.1198, R^{2}=.01436, p=.8211 \\
\text { Combined ABA: } r=.1201, R^{2}=.01442, p=.7101\end{array}$ & IL-4: ABA-S n=6, ABA-R n=6, Combined ABA $n=12$ \\
\hline
\end{tabular}


$1 \mathrm{~A}$

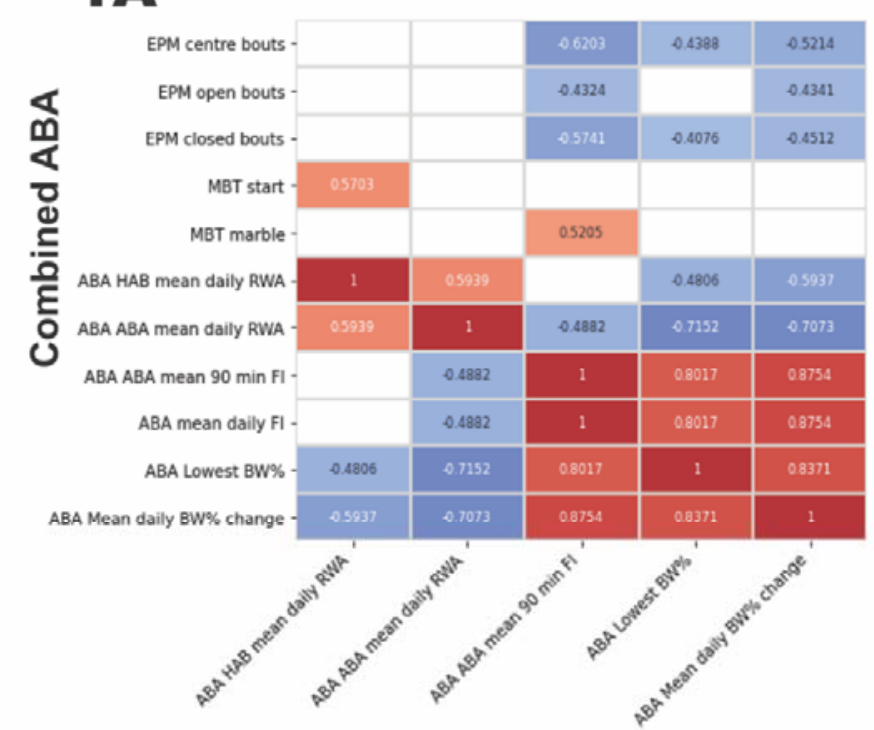

$1 B$

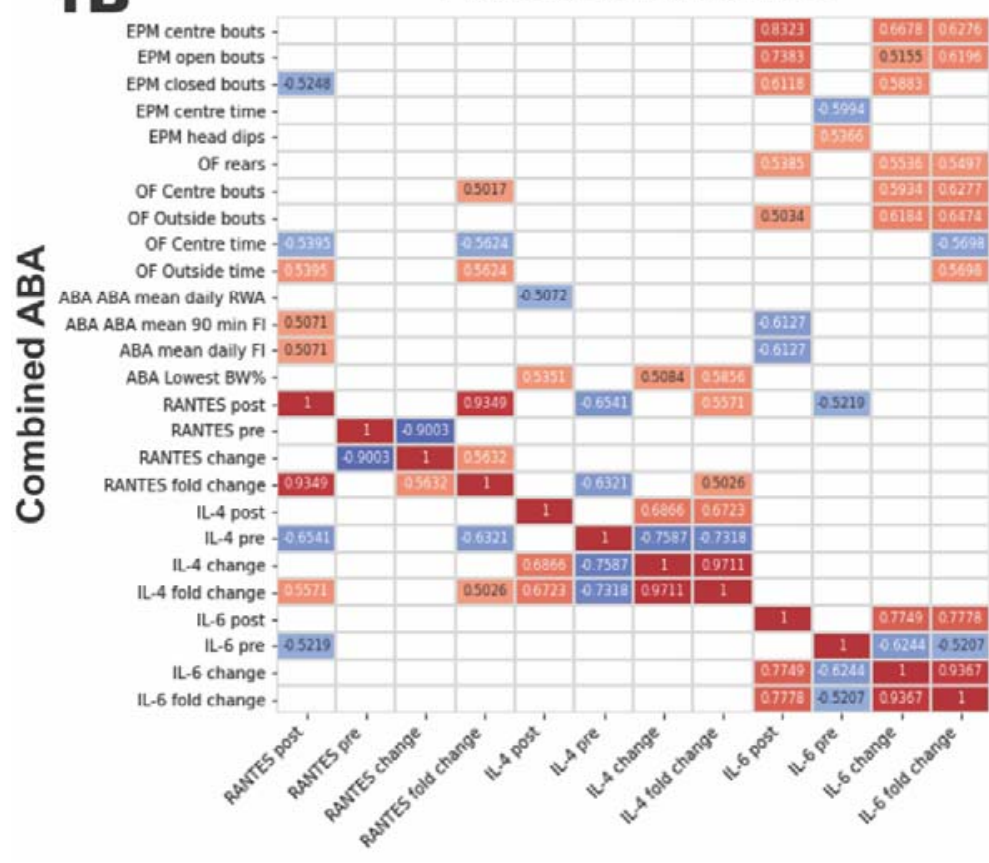

\section{Corresponding $\mathbf{P}$ values}

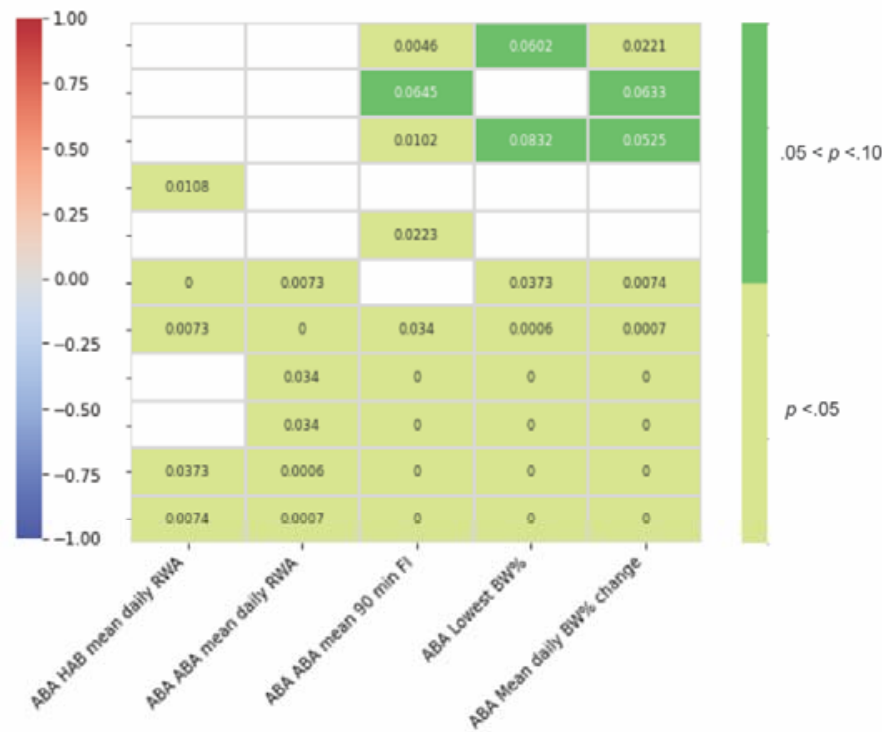

\section{Corresponding $\mathbf{P}$ values}

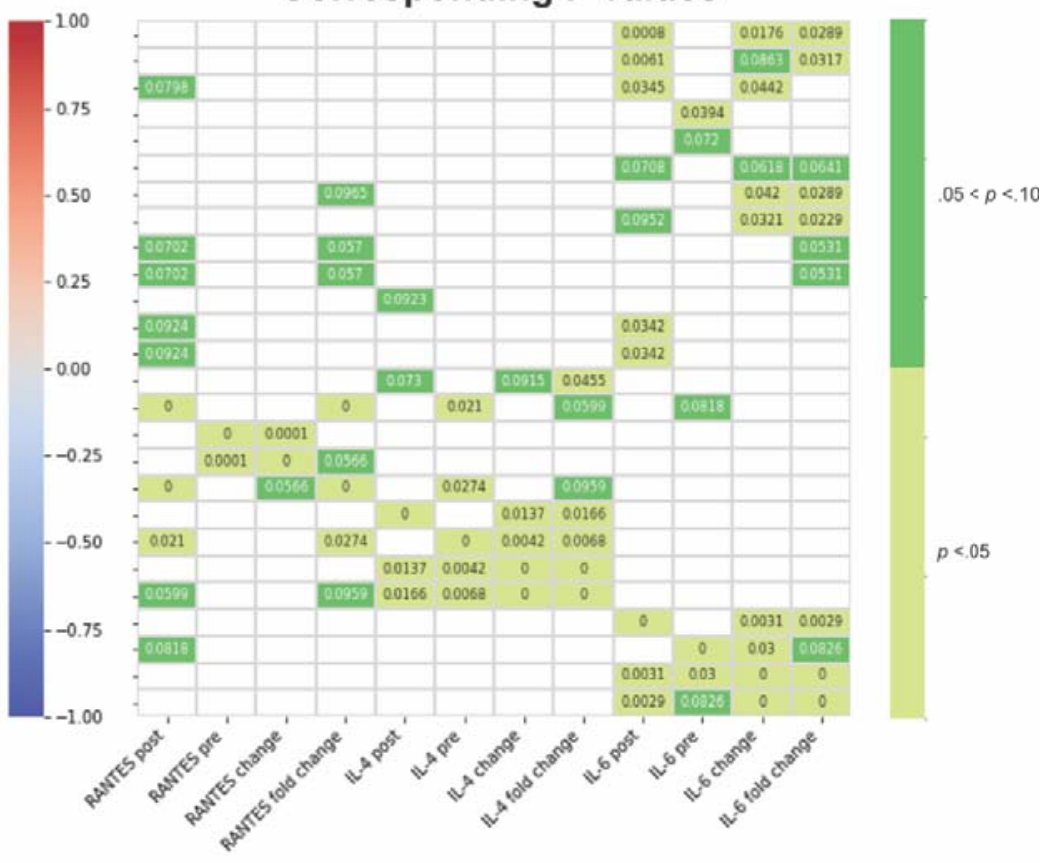

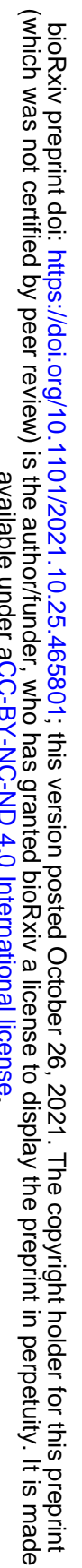


2A
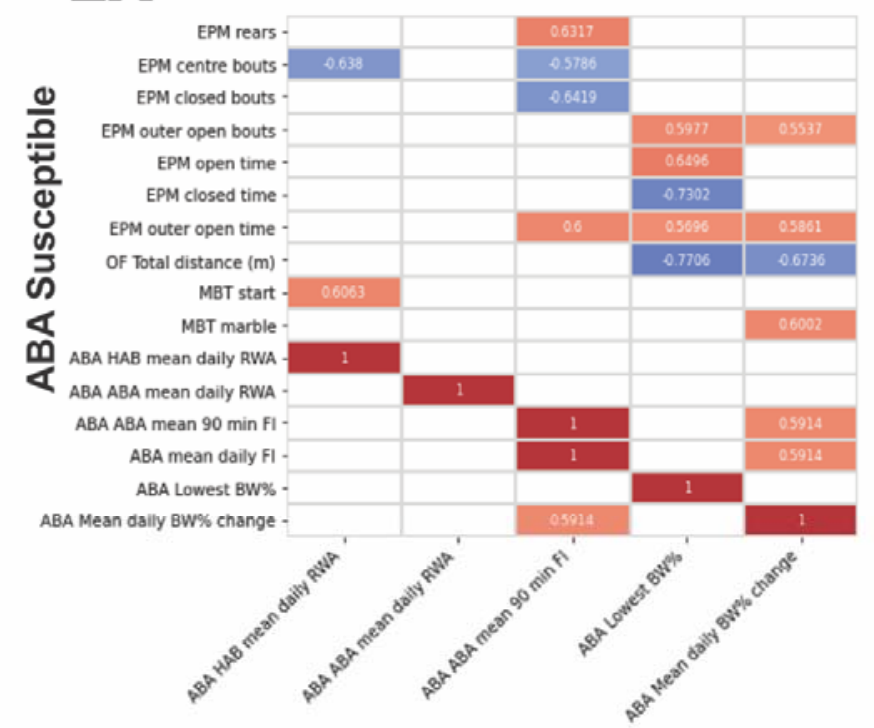

2B

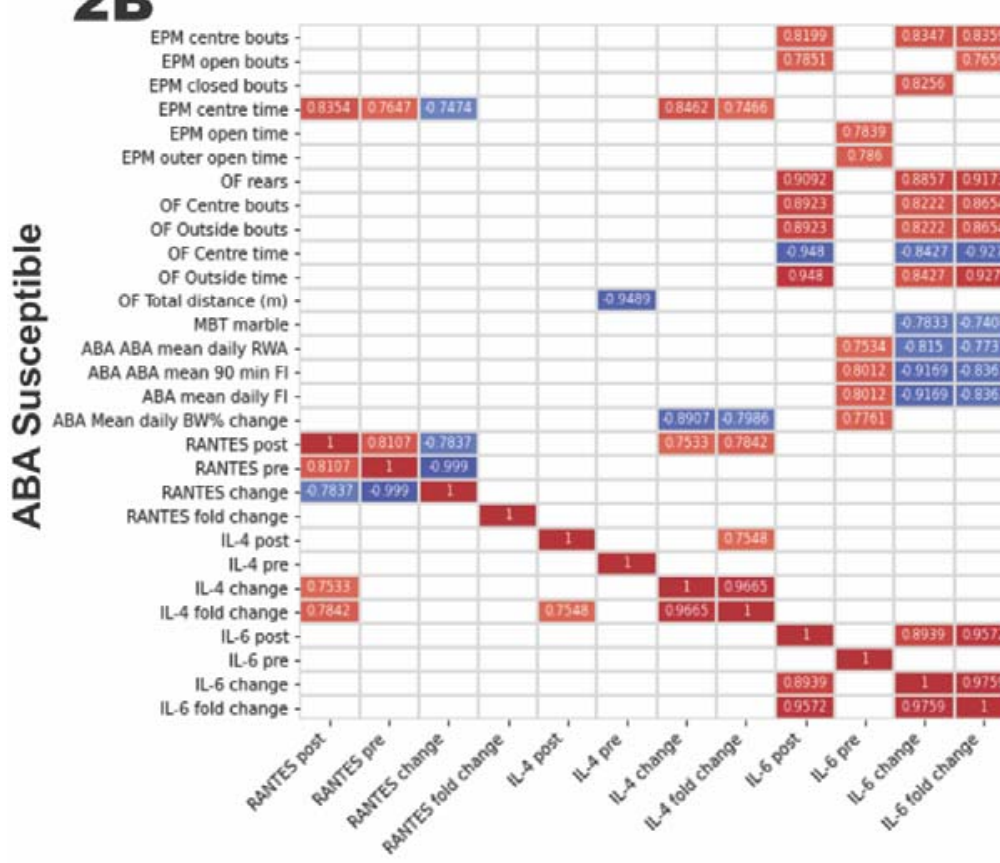

\section{Corresponding $\mathbf{P}$ values}

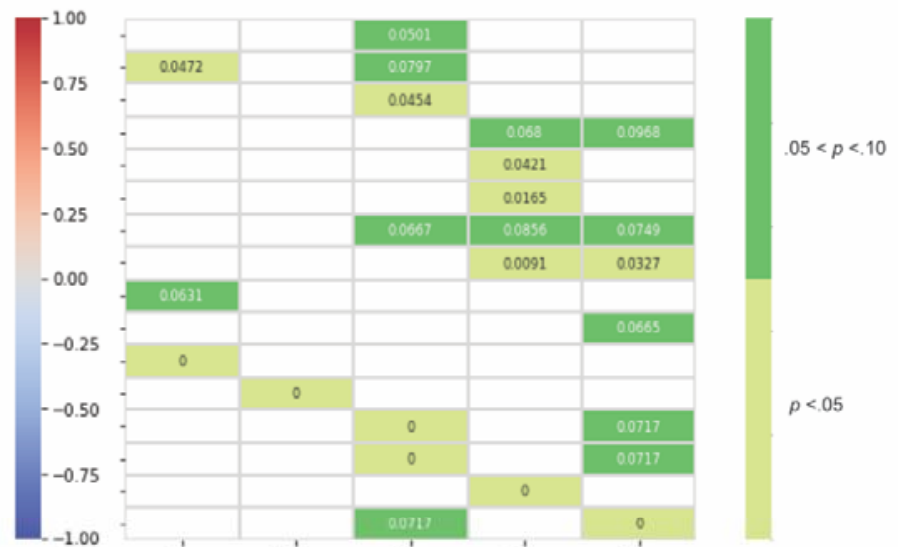

\section{Corresponding $\mathrm{P}$ values}

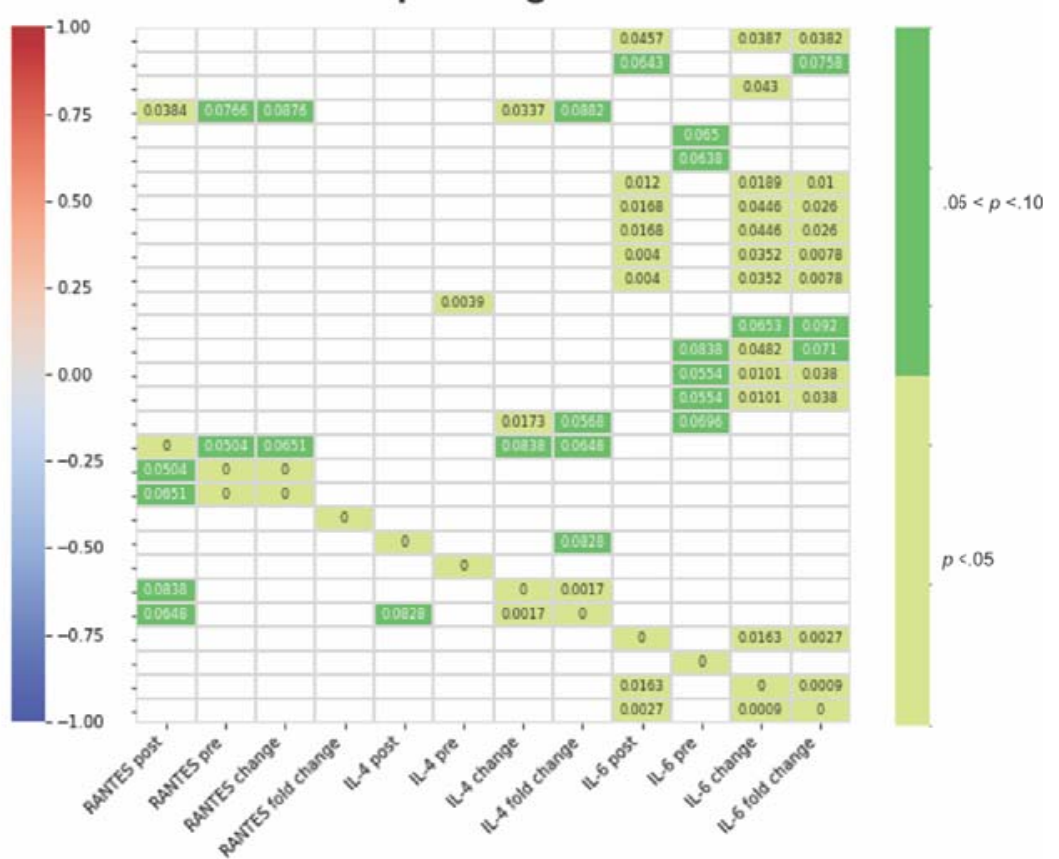




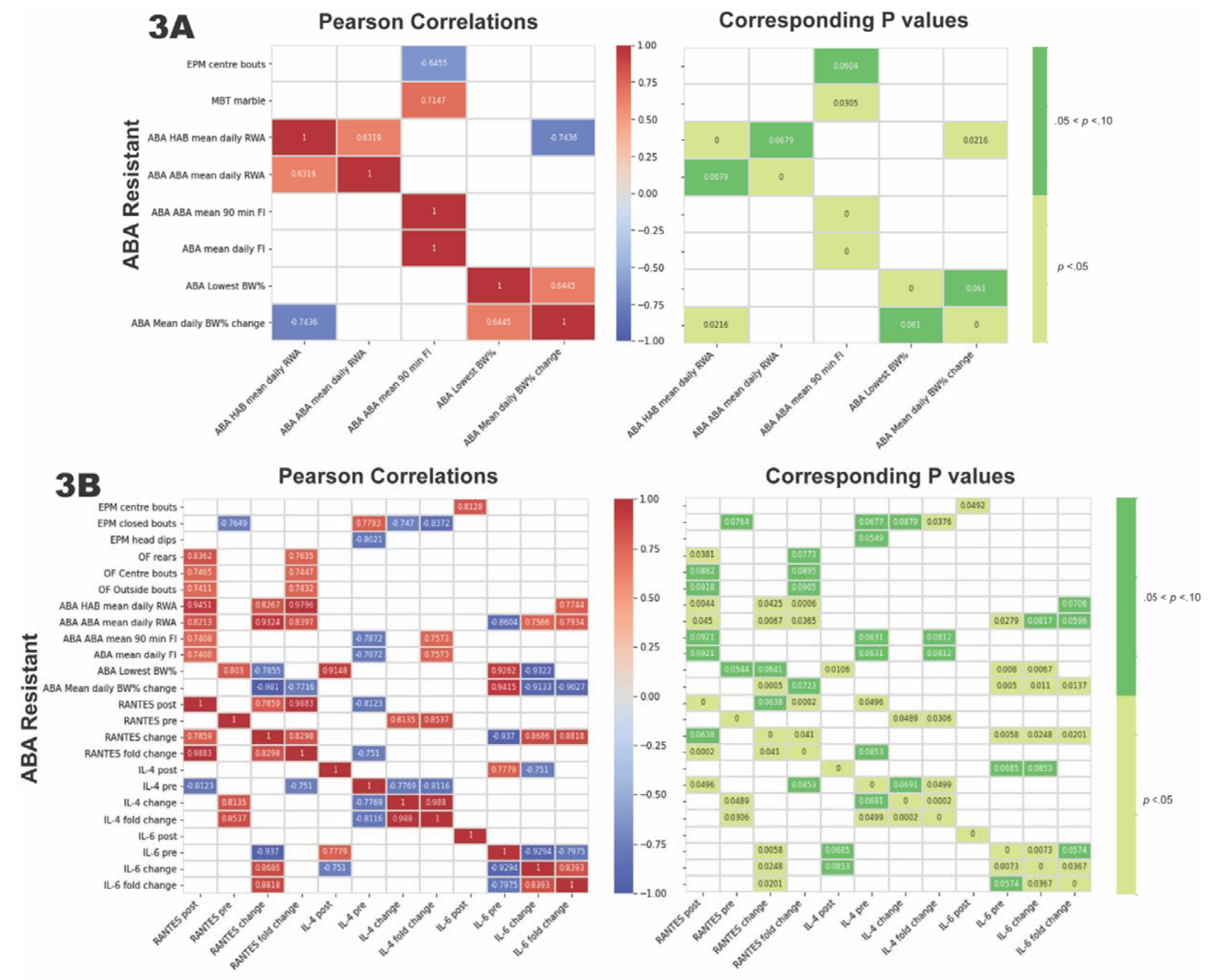

\section{Corresponding $\mathrm{P}$ values}


Supplementary Figures 1-3. For each of the 1) Combined ABA, 2) ABA Susceptible, and 3) ABA Resistant groups, Python was used to generate Pearson's correlation and corresponding $p$ value matrices for $\mathbf{A}$ ) outcomes from behavioural tests prior to ABA vs. ABA outcome measures, and $\mathbf{B}$ ) outcomes from behavioural tests prior to ABA and ABA outcome measures vs. cytokine levels (pre-exposure, post-exposure, absolute change and fold-change from pre- to post-exposure). Python was then utilized to produce the above plots of these matrices showing data only for pairs of variables where $p<.01$.

Group sizes for behavioural correlations: Combined ABA $n=19$; ABA-S $n=10$; ABA-R $n=9$ and for cytokine correlations: Combined ABA $n=12$; ABA-S $n=6$; ABA-R $n=6$. Abbreviations: EPM, elevated plus maze; OF, open field; bouts, number of entries into zone; time, percentage of total 10-minute test duration, MBT, marble burying test; start, latency in seconds until first episode of digging; marbles, number of interactions with marbles; $A B A$, activity-based anorexia; $A B A H A B$, baseline phase of $A B A ; A B A$ ABA, ABA (food restriction) period of ABA; RWA, running wheel activity; FI, food intake; BW\%, body weight \%. 